\title{
Star-formation laws in luminous infrared galaxies
}

\section{New observational constraints on models ${ }^{\star}$}

\author{
S. García-Burillo ${ }^{1}$, A. Usero ${ }^{1}$, A. Alonso-Herrero ${ }^{2,3}$, J. Graciá-Carpio ${ }^{4}$, M. Pereira-Santaella ${ }^{2}$, \\ L. Colina ${ }^{2}$, P. Planesas ${ }^{1,5}$, and S. Arribas ${ }^{2}$
}

\author{
1 Observatorio Astronómico Nacional (OAN)-Observatorio de Madrid, Alfonso XII, 3, 28014 Madrid, Spain \\ e-mail: s.gburillo@oan.es \\ 2 Centro de Astrobiología (CSIC-INTA), Ctra de Torrejón a Ajalvir, km 4, 28850 Torrejón de Ardoz, Madrid, Spain \\ 3 Instituto de Física de Cantabria, CSIC-UC, Avenida de los Castros s/n, 39005 Santander, Spain \\ ${ }^{4}$ Max-Planck-Institut für extraterrestrische Physik, Giessenbachstrasse 1, 85748 Garching, Germany \\ 5 ESO \& Joint ALMA Observatory, Alonso de Cordova 3107, Vitacura, Santiago, Chile
}

Received 5 August 2011 / Accepted 28 November 2011

\begin{abstract}
Context. The observational study of star-formation relations in galaxies is central for unraveling the related physical processes that are at work on local and global scales. It is still debated whether star formation can be described by a universal law that remains valid in different populations of galaxies.

Aims. We aim to expand the sample of extreme starbursts, represented by local luminous and ultra-luminous infrared galaxies (LIRGs and ULIRGs), with high-quality observations in the 1-0 line of $\mathrm{HCN}$, which is taken as a proxy for the dense molecular gas content. The new data presented in this work allow us to enlarge in particular the number of LIRGs studied in HCN by a factor 3 compared to previous works. The chosen LIRG sample has a range of HCN luminosities that partly overlaps with that of the normal galaxy population. We study if a universal law can account for the star-formation relations observed for the dense molecular gas in normal star-forming galaxies and extreme starbursts and explore the validity of different theoretical prescriptions of the star-formation law. Methods. We have used the IRAM $30 \mathrm{~m}$ telescope to observe a sample of 19 LIRGs in the $1-0$ lines of CO, $\mathrm{HCN}$ and $\mathrm{HCO}^{+}$. The galaxies were extracted from a sample of local LIRGs with available high-quality and high-resolution images obtained at optical, near and mid IR wavelengths, which probe the star-formation activity. We therefore derived the star-formation rates using different tracers and determined the sizes of the star-forming regions of all targets.

Results. The analysis of the new data proves that the efficiency of star formation in the dense molecular gas ( $\mathrm{SFE}_{\text {dense }}$ ) of extreme starbursts is a factor 3-4 higher compared to normal galaxies. Kennicutt-Schmidt (KS) power laws were also derived. We find a duality in KS laws that is further reinforced if we account for the likely different conversion factor for $\mathrm{HCN}\left(\alpha^{\mathrm{HCN}}\right)$ in extreme starbursts and for the unobscured star-formation rate in normal galaxies. This result extends the more extreme bimodal behavior of star-formation laws that was derived from CO molecular lines by two recent surveys to the higher molecular densities probed by HCN lines.

Conclusions. We compared our observations with the predictions of theoretical models in which the efficiency of star formation is determined by the ratio of a constant star-formation rate per free-fall time $\left(S F R_{\mathrm{ff}}\right)$ to the local free-fall time $\left(t_{\mathrm{ff}}\right)$. We find that it is possible to fit the observed differences in the $\mathrm{SFE}_{\text {dense }}$ between normal galaxies and LIRGs/ULIRGs using a common constant $S F R_{\mathrm{ff}}$ and a set of physically acceptable HCN densities, but only if $S F R_{\mathrm{ff}} \sim 0.005-0.01$ and/or if $\alpha^{\mathrm{HCN}}$ is a factor of $\sim$ a few lower than our favored values. Star-formation recipes that explicitly depend on the galaxy global dynamical time scales do not significantly improve the fit to the new HCN data presented in this work.
\end{abstract}

Key words. galaxies: ISM - radio lines: ISM - galaxies: starburst - galaxies: star formation - radio lines: galaxies

\section{Introduction}

The quest for a quantitative description of the star-formation rate dependence on a set of galaxy parameters (gas and stellar content, gas kinematics, environment) is a fundamental, though not fully understood problem. Observational constraints are central to understanding the role that small-scale (local) and large-scale (global) galaxy properties play in star-formation relations. This is a requisite to validating the recipes of star formation used in numerical simulations of galaxy evolution.

Based on simple theoretical grounds, Schmidt (1959) proposed that the star-formation rate (SFR) per unit volume $\left(\rho_{\mathrm{SFR}}\right)$

\footnotetext{
* Based on observations carried out with the IRAM $30 \mathrm{~m}$ telescope. IRAM is supported by INSU/CNRS (France), MPG (Germany) and IGN (Spain).
}

should be a power law of index $N$ of the gas volume density $\left(\rho_{\text {gas }}\right): \rho_{\mathrm{SFR}} \propto \rho_{\text {gas }}^{N}$. Schmidt (1959) predicted that $N$ should be around 2. If we suppose that the relevant time scale for star formation is the local free-fall time $\left(t_{\mathrm{ff}}\right)$, in this case $\rho_{\mathrm{SFR}} \propto$ $\rho_{\text {gas }} / t_{\mathrm{ff}} \propto \rho_{\text {gas }}^{1.5}$. If the gas scale-height is constant among galaxies, a non-trivial additional assumption, the equivalent of the Schmidt law in terms of the corresponding surface densities of $\operatorname{SFR}\left(\Sigma_{\mathrm{SFR}}\right)$ and gas $\left(\Sigma_{\text {gas }}\right)$ is $\Sigma_{\mathrm{SFR}} \propto \Sigma_{\text {gas }}^{1.5}$.

Krumholz \& McKee (2005, see also Krumholz et al. 2009) developed a model that successfully describes how star formation takes place in a highly turbulent molecular medium. Their scenario pictures star formation as a very inefficient process that takes place in small subregions of supersonic turbulent and virialized molecular clouds. The surface density of the star-formation rate is in any case expected to scale as a power 
law of the mean density of the gas with an index close to 1.5 . Star formation in Krumholz \& McKee's formulation is regulated by processes mostly within the clouds. The relevant time scale for these models, the free-fall time for cloud collapse, consequently is a local time scale. In this type of formulation, star-formation laws should not depend explicitly on global dynamical time scales or be determined by galactic-scale phenomena. Instead, large-scale processes are thought to be responsible for making the gas assembly prior to star formation in galaxies. Alternatively, other forms of the star-formation laws adduce the importance of including explicitly global dynamical time scales $t_{\text {dyn }}$ (Elmegreen 1997; Silk 1997; Kennicutt 1998; Elmegreen 2002; Boissier et al. 2003; Tasker \& Tan 2009; Tan 2010; Genzel et al. 2010). The star-formation rate density $\Sigma_{\mathrm{SFR}}$ should then scale as $\propto \Sigma_{\text {gas }} / t_{\mathrm{dyn}}$. It is unclear as yet if the two forms of star-formation laws are fully equivalent in normal galaxies and in mergers. Kennicutt (1998) found that if $t_{\mathrm{dyn}}$ is the average orbital dynamical time scale, both formulations are equally successful at describing star-formation laws in the disks of normal galaxies. The physical framework for this equivalence relies on the fact that normal galaxy disks, viewed as dynamically relaxed stable systems, fulfill the Toomre stability criterion (Toomre 1964). It is questionable, nevertheless, if this equivalence should also hold in extreme starbursts, which are often hosted by merging systems.

During the last decades observers have been using different proxies for $\Sigma_{\mathrm{SFR}}$ and $\Sigma_{\text {gas }}$ to validate the predictions of star-formation law models. These observations have been conducted on different galaxy samples, including normal galaxies and extreme starbursts at different redshifts. They also encompass a wide range of spatial resolutions, which allow not only global measurements but also studies of spatially resolved objects. The different observers do not find a single exponent fitting the entire star-formation relation. Kennicutt (1998) finds an index $n=1.40 \pm 0.15$ using $\mathrm{CO}$ and $\mathrm{HI}$ as gas tracers and a $~ 100$ galaxy sample that includes normal galaxies, nuclear starbursts and a few luminous infrared galaxies (LIRGs) $(\sim 5)$ at $z=0$. Yao et al. (2003) find an index $n=1.40 \pm 0.30$ in their CO survey of IR luminous galaxies. Bouché et al. (2007), by including submillimeter galaxies (SMGs) and using a lower $\mathrm{CO}$ conversion factor in $z=0$ ultra-luminous infrared galaxies (ULIRGs), determines a higher index for the Kennicutt-Schmidt (KS) law $n \sim 1.7$. The high-resolution survey of 18 normal galaxies published by Bigiel et al. (2008) and Leroy et al. (2008) found a linear KS relation above a gas surface density threshold of $10 M_{\odot} \mathrm{pc}^{-2}$, which determines the transition from atomic to molecular gas, here traced by $\mathrm{CO}$ lines.

More recently the CO surveys of Daddi et al. (2010) and Genzel et al. (2010) presented evidence that normal galaxies and mergers (LIRGs, ULIRGs and SMGs) occupy different regions in the molecular gas mass versus star-formation rate plane. These results suggest the existence of a bimodality in star-formation laws, where normal galaxies show 4-10 longer depletion time-scales compared to mergers. While the KS relation remains mostly linear inside each galaxy population, bimodality introduces a discontinuity in the two-function power law. These authors discussed that when global dynamical time scales are included, a universal star-formation law is obtained. Based on a study of far-infrared fine structure lines, Graciá-Carpio et al. (2011) concluded that extreme starbursts display significantly enhanced line deficits. The results of Graciá-Carpio et al. (2011) provide additional support for the existence of different properties of the interstellar medium in normal star-forming galaxies and extreme starburst systems.
Krumholz \& Thompson (2007; see also Narayanan et al. 2008) studied how the power index of KS laws determined from observations should change depending on the critical density of the tracer used to probe the star-forming gas, a prediction confirmed by observations conducted in several dense gas tracers (Narayanan et al. 2005; Bussmann et al. 2008; Bayet et al. 2009; Juneau et al. 2009). In the particular case of $\operatorname{HCN}(1-0)$, only in galaxies where the average gas density exceeds a few $10^{4} \mathrm{~cm}^{-3}$ (i.e., the effective critical density of the $\mathrm{HCN} J=1-0$ line), we would start to recover the expected superlinear behavior of the universal KS law derived by Krumholz \& McKee (2005).

Observations of HCN lines have been used to study dense molecular gas properties in samples of normal spirals, LIRGs, ULIRGs, quasi-stellar objects (QSOs) and molecular gas-rich high- $z$ galaxies (e.g., Gao \& Solomon 2004a,b; Solomon \& Vanden Bout 2005; Wagg et al. 2005; Riechers et al. 2007; Graciá-Carpio et al. 2006, 2008). In contrast to CO line observations, which are sensitive to the global molecular gas content, $\mathrm{HCN}$ observations are a much better tracer of the dense molecular phase, which is more directly related to star formation, and can therefore put stringent constraints on star-formation models. The most important result presented by Gao \& Solomon in their seminal papers is the discovery of a tight correlation between the infrared and the $\mathrm{HCN}(1-0)$ luminosities over three orders of magnitude in $L_{\mathrm{IR}}$. This result was interpreted as evidence that star formation is the main power source in ULIRGs. The linearity of the tight correlation between the IR and the $\mathrm{HCN}(1-0)$ luminosities implies that the star-formation efficiency measured with respect to the dense molecular gas content $\left(\mathrm{SFE}_{\text {dense }} \propto\right.$ $\left.L_{\mathrm{IR}} / L_{\mathrm{HCN}(1-0)}^{\prime}\right)$ is constant in all galaxies, independently of $L_{\mathrm{IR}}$.

More recent results have partly questioned this picture, however. Graciá-Carpio et al. (2008) published observations made with the IRAM $30 \mathrm{~m}$ telescope of the $J=1-0$ and 3-2 lines of $\mathrm{HCN}$ and $\mathrm{HCO}^{+}$used to probe the dense molecular gas content of a sample of 17 LIRGs and ULIRGs. These observations were also used to derive a new version of the power law describing the correlation between $L_{\mathrm{FIR}}$ and $L_{\mathrm{HCN}(1-0)}^{\prime}$ from normal galaxies $\left(L_{\mathrm{IR}}<10^{11} L_{\odot}\right)$ to high- $z$ galaxies. The results of Graciá-Carpio et al. (2008) indicated that the $L_{\mathrm{FIR}} / L_{\mathrm{HCN}(1-0)}^{\prime}$ ratio, taken as proxy for $\mathrm{SFE}_{\text {dense }}$, is a factor $\sim 2-3$ higher in IR luminous targets compared to normal galaxies. Furthermore, Graciá-Carpio et al. (2008) found, based on a multiline analysis of $\mathrm{HCN}$ and $\mathrm{HCO}^{+}$data, that $X_{\mathrm{HCN}}$ is probably about three times lower at high $L_{\mathrm{FIR}}$. Taken together, these findings suggest that $\mathrm{SFE}_{\mathrm{dense}}$ may be up to an order of magnitude higher in extreme starbursts (LIRGs, ULIRGs) than in normal galaxies.

Compared to normal galaxies and the most extreme starbursts represented by ULIRGs, LIRGs can constitute the transition point in the star-formation laws of galaxies. The main purpose of this paper is to enlarge the sample of LIRGs with high-quality $\mathrm{HCN}(1-0)$ observations. This is a key requirement for testing if the observed bimodality of star-formation laws in galaxies derived from $\mathrm{CO}$ can be extended to the higher density regime probed by $\mathrm{HCN}$. We used these results to explore the validity of different star-formation law theoretical prescriptions that are currently debated. To this aim we have used the $30 \mathrm{~m}$ telescope to observe a sample of 19 LIRGs in the 1-0 lines of $\mathrm{CO}, \mathrm{HCN}$ and $\mathrm{HCO}^{+}$. Most of the targets were extracted from the complete sample of local $(d<78 \mathrm{Mpc})$ LIRGs studied by Alonso-Herrero et al. (2006). All targets in this sample have high-quality and high-resolution imaging at optical, near infrared (NIR) and mid infrared (MIR) wavelengths (obtained with HST, VLT, CAHA and Spitzer). This allows us to 
accurately derive the sizes of the star-forming regions and the star-formation rates for all the targets. The new data presented in this work allow us to expand the number of LIRGs studied in $\mathrm{HCN}-\mathrm{HCO}^{+}$lines by more than a factor 3 compared to previous works (nine LIRGs in Graciá-Carpio et al.'s sample).

\section{The sample of LIRGs}

We obtained new observations for a sample of 19 LIRGs. Out of these, fourteen were extracted from the sample of nearby LIRGs studied by Alonso-Herrero et al. (2006). This is a volume-limited sample $\left(v<5200 \mathrm{~km} \mathrm{~s}^{-1}\right.$, distances of between 35 and $\left.78 \mathrm{Mpc}\right)$ drawn from the IRAS Revised Bright Galaxy Sample (RBGS) analyzed by Sanders et al. (2003). Alonso-Herrero et al. (2006) required the logarithm of the total IR luminosity to be $\geq 11.05 L_{\odot}$ and the galaxies to be at Galactic latitude $b>10^{\circ}$. The remaining five LIRGs were selected to populate the high end of the IR luminosity distribution of LIRGs. For the AlonsoHerrero et al. LIRGs we obtained HST/NICMOS Pa $\alpha$ imaging (Alonso-Herrero et al. 2001, 2002, 2006), as well as groundbased optical integral field spectroscopy using CAHA/PMAS and VLT/VIMOS covering the $\mathrm{H} \alpha$ spectral region (AlonsoHerrero et al. 2009; Rodríguez Zaurín et al. 2011). In addition there are MIR Spitzer imaging observations for the entire sample from the GOALS sample (Armus et al. 2009). This means that the SFRs of the sample are very well characterized from hydrogen recombination lines $(\mathrm{H} \alpha, \mathrm{Pa} \alpha)$ and MIR $(24 \mu \mathrm{m})$ luminosities on the same angular scales probed by the IRAM observations. We also have a good estimate of the typical size of the star-forming regions in all galaxies of our sample, thanks to the available high-resolution imaging $(\mathrm{H} \alpha, \mathrm{Pa} \alpha)$. This minimizes the errors when both $\Sigma_{\mathrm{SFR}}$ and $\Sigma_{\text {dense }}$, required to derive $\mathrm{KS}$ laws, are evaluated. The estimated sizes are typically $<10^{\prime \prime}$ in all sources, equivalent to $1.7-3.6 \mathrm{kpc}$ for the given range of distances. This guarantees that the total emission of the molecular gas is contained in the $30 \mathrm{~m}$ beam $\left(\sim 28^{\prime \prime}\right.$ at the frequency of the $\mathrm{HCN}(1-0)$ line).

Additionally, we used a compiled sample of 108 galaxies with published far infrared (FIR) and $\mathrm{HCN}(1-0)$ observations in the literature. When available, we also included $\mathrm{HCO}^{+}(1-$ 0 ) data. In addition to the new data obtained in LIRGs for this work we include the data of normal galaxies, LIRGs and ULIRGs of Gao \& Solomon (2004a), data from the sample of infrared-excess Palomar-Green QSOs (Evans et al. 2006) and data from high- $z$ galaxies with available $\mathrm{HCN}$ observations (see Gao et al 2007, and references therein). Following the same approach as adopted by Graciá-Carpio et al. (2008), in those high$z$ sources where $\mathrm{HCN}(1-0)$ are not available, $L_{\mathrm{HCN}(1-0)}^{\prime}$ was derived assuming that their rotational line luminosity ratios are $L_{\mathrm{HCN}(2-1)}^{\prime} / L_{\mathrm{HCN}(1-0)}^{\prime}=0.7$, similar to the mean value measured by Krips et al. (2008). For the $z \simeq 4$ quasar APM 08279+5255 we adopted $L_{\mathrm{HCN}(5-4)}^{\prime} / L_{\mathrm{HCN}(1-0)}^{\prime}=0.3$, assuming the physical conditions derived in García-Burillo et al. (2006).

\section{Observations}

The new molecular line observations of the sample of 19 LIRGs described in Sect. 2 were carried out in one observing run in June 2008 with the IRAM 30 m telescope at Pico de Veleta (Spain). We tuned the $3 \mathrm{~mm}$ and $1 \mathrm{~mm}$ SIS receivers of the $30 \mathrm{~m}$ telescope to the redshifted frequencies of the $\mathrm{HCN}(1-0)$, $\mathrm{HCO}^{+}(1-0), \mathrm{CO}(1-0)$ and $\mathrm{CO}(2-1)$ lines. The beam sizes of the $30 \mathrm{~m}$ telescope range from $F W H M \sim 28^{\prime \prime}$ at $88 \mathrm{GHz}$ to $\sim 11^{\prime \prime}$ at $230 \mathrm{GHz}$ ). We covered a velocity range $1300-1800 \mathrm{~km} \mathrm{~s}^{-1}$ for the $3 \mathrm{~mm}$ lines and $1200 \mathrm{~km} \mathrm{~s}^{-1}$ for the $1 \mathrm{~mm}$ lines. We used the wobbler switching mode to make baselines flatter. System temperatures during the observations were typically $\sim 100-130 \mathrm{~K}$ at $3 \mathrm{~mm}$ and $\sim 300-500 \mathrm{~K}$ at $1 \mathrm{~mm}$. Receivers were used in single side-band mode (SSB), with a high rejection of the image band: $>12 \mathrm{~dB}$ at $1 \mathrm{~mm}$ and $>20 \mathrm{~dB}$ at $3 \mathrm{~mm}$. The calibration accuracy is estimated to be better than $20 \%$. We checked the pointing of the telescope every $1.5 \mathrm{~h}$ by observing nearby continuum sources; the average rms pointing error was $2^{\prime \prime}$ during the observing run.

Throughout the paper, velocity-integrated line intensities $(I)$ are given in antenna temperature scale, $T_{\mathrm{a}}^{*}$. The $T_{\mathrm{a}}^{*}$ scale relates to the main beam temperature scale, $T_{\mathrm{mb}}$, by the equation $T_{\mathrm{mb}}=$ $\left(F_{\text {eff }} / B_{\text {eff }}\right) T_{\mathrm{a}}^{*}$, where $F_{\text {eff }}$ and $B_{\text {eff }}$ are the forward and beam efficiencies of the telescope at a given frequency. For the IRAM $30 \mathrm{~m}$ telescope $F_{\text {eff }} / B_{\text {eff }}=1.22(1.57)$ at $87-88 \mathrm{GHz}(230 \mathrm{GHz})$ and $S / T_{\mathrm{mb}}=4.95 \mathrm{Jy} \mathrm{K}^{-1}$. The velocity windows used to derive $I_{\mathrm{HCO}^{+}}$and $I_{\mathrm{HCN}}$ were defined from the corresponding higher signal-to-noise CO line profiles. We subtracted linear baselines to the individual scans and eliminated those showing instabilities requiring higher order baselines. We show in Fig. 1 the ensemble of $\mathrm{HCN}(1-0), \mathrm{HCO}^{+}(1-0)$ and $\mathrm{CO}(1-0)$ spectra used in this work. Molecular line luminosities $\left(L^{\prime}\right)$ were computed in units of $L^{\prime}=\mathrm{K} \mathrm{km} \mathrm{s}^{-1} \mathrm{pc}^{2}$ as defined by Gao \& Solomon (2004a). Luminosity distances were derived assuming a flat $\Lambda$-dominated cosmology described by $H_{0}=71 \mathrm{~km} \mathrm{~s}^{-1} \mathrm{Mpc}^{-1}$ and $\Omega_{\mathrm{m}}=0.27$. Observational parameters and results are summarized in Tables 1 and 2 .

\section{Star-formation laws of the dense gas}

In Sects. 4.1 and 4.2 we study the star-formation efficiency of the dense gas $\left(\mathrm{SFE}_{\text {dense }}\right)$ and the KS laws in the different populations of galaxies compiled for this work, including the new sample of LIRGs. Following the same approach as adopted by Graciá-Carpio et al. (2008), we derive an SFR $\left[M_{\odot} \mathrm{yr}^{-1}\right]$ from $L_{\mathrm{IR}}^{\mathrm{SFR}}$ defined as $L_{\mathrm{IR}}^{\mathrm{SFR}}=1.3 \times L_{\mathrm{FIR}}(40-500 \mu \mathrm{m})$. This aims at minimizing the possible active galactic nucleus (AGN) contribution to $L_{\mathrm{IR}}(8-1000 \mu \mathrm{m})$. The $\mathrm{AGN}$ contribution to $L_{\mathrm{IR}}$ in the population of local LIRGs with AGN detections is nonetheless known to be small, with a median value of $L_{\mathrm{bol}}(\mathrm{AGN}) / L_{\mathrm{IR}}=0.05$ (Alonso-Herrero et al. 2012).

In this section we adopt the common assumptions regarding conversion factors used to derive SFR from $L_{\mathrm{IR}}^{\mathrm{SFR}}$ and $M_{\text {dense }}$ from $L_{\mathrm{HCN}(1-0)}^{\prime}$. We first adopt the same universal factor used by Kennicutt (1998):

$\operatorname{SFR}\left[M_{\odot} \mathrm{yr}^{-1}\right]=1.7 \times 10^{-10} L_{\mathrm{IR}}^{\mathrm{SFR}}\left[L_{\odot}\right]$.

Similarly, $M_{\text {dense }}$ is obtained in the following from $L_{\mathrm{HCN}(1-0)}^{\prime}$ assuming a universal conversion factor $\left(\alpha^{\mathrm{HCN}}\right)$ similar to that of Gao \& Solomon (2004a):

$M_{\text {dense }}\left[M_{\odot}\right]=10 L_{\mathrm{HCN}(1-0)}^{\prime}\left[\mathrm{K} \mathrm{km} \mathrm{s}^{-1} \mathrm{pc}^{2}\right]$.

We nevertheless question the grounds for these two working assumptions and their consequences in Sect. 6.

\subsection{Star-formation efficiency (SFE $\left.E_{\text {dense }}\right)$}

Figure 2 shows the $L_{\mathrm{FIR}} / L_{\mathrm{HCN}(1-0)}^{\prime}$ luminosity ratio versus $L_{\mathrm{FIR}}$ for the different populations of galaxies in this compilation, defined in Sect. 2. We adopt here the common assumption that the $L_{\mathrm{FIR}} / L_{\mathrm{HCN}(1-0)}^{\prime}$ luminosity ratio is a good proxy for 


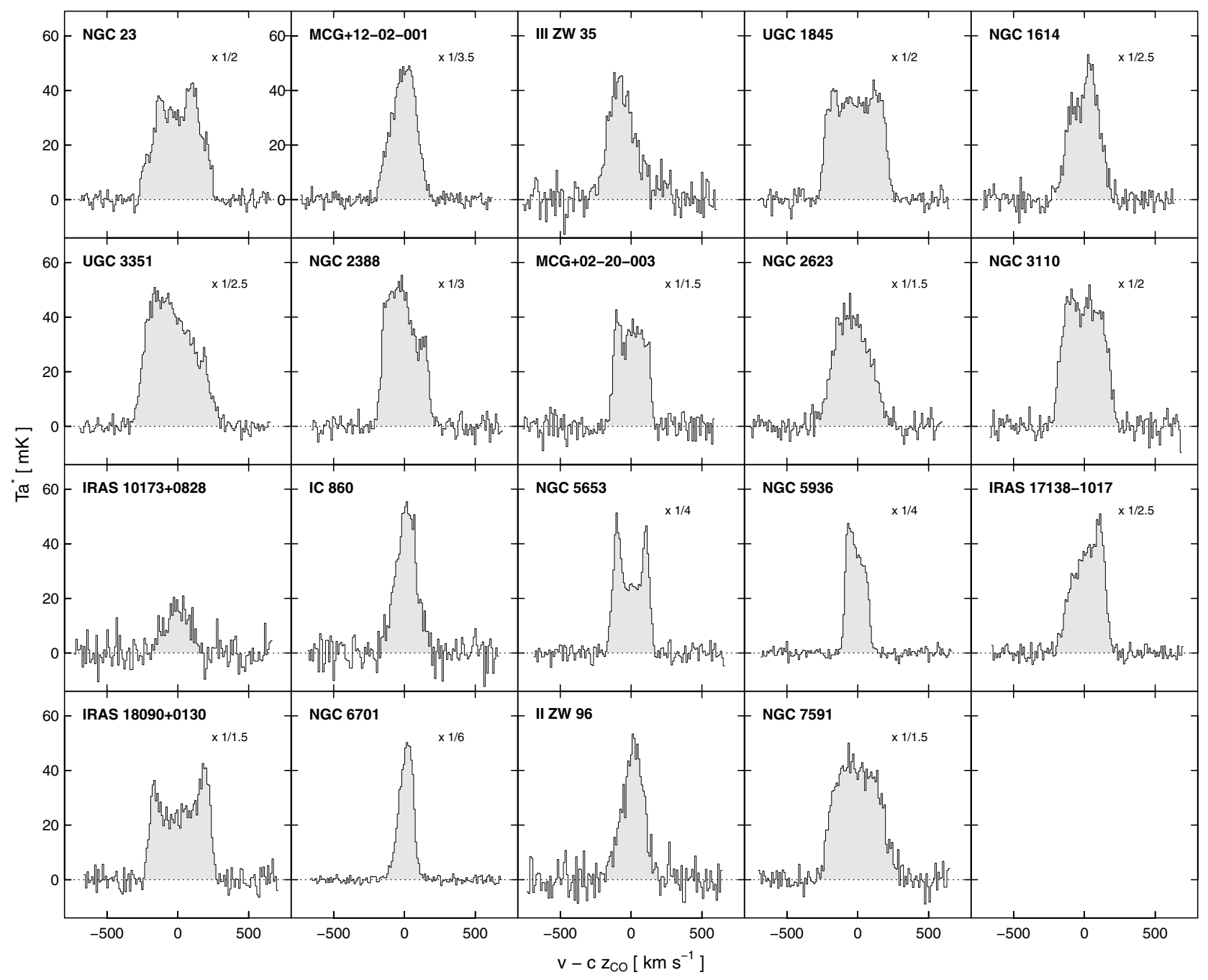

Fig. 1. a) $C O(1-0)$ spectra observed with the IRAM $30 \mathrm{~m}$ telescope in a sample of 19 LIRGs. Spectra appear from top to bottom and from left to right in order of increasing right ascension of the source. For clarity some of the line intensities were scaled by the factor indicated in the panel. Gray-filled histograms highlight the velocity range used to calculate the baseline fitting and the line areas.

the star-formation efficiency of the dense gas $\left(\mathrm{SFE}_{\text {dense }} \equiv\right.$ $\left.\mathrm{SFR} / M_{\text {dense }} \propto L_{\mathrm{FIR}} / L_{\mathrm{HCN}(1-0)}^{\prime}\right)$. We estimate that the total uncertainty on both $L_{\mathrm{FIR}}$ and $L_{\mathrm{HCN}(1-0)}^{\prime}$ (and related quantities), with an equal contribution of statistical and systematic errors, amounts to $\pm 30 \%( \pm 0.13 \mathrm{dex})$. The corresponding total uncertainty on $L_{\mathrm{IR}} / L_{\mathrm{HCN}(1-0)}^{\prime}$ is $\pm 42 \%( \pm 0.19 \mathrm{dex})$.

In Fig. 2 we can see that $S_{\text {dense }}$ increases with $L_{\text {FIR }}$ from normal galaxies to extreme starbursts, represented by LIRGs, ULIRGs and high- $z$ objects. In particular, the orthogonal regression fit to the full sample of objects gives a power law index for $\mathrm{SFE}_{\text {dense }}$ significantly different from zero:

$\log \left(\frac{L_{\mathrm{FIR}}}{L_{\mathrm{HCN}(1-0)}^{\prime}}\right)=(0.29 \pm 0.04) \log L_{\mathrm{FIR}}-(0.23 \mp 0.42)$

or $\frac{L_{\mathrm{FIR}}}{L_{\mathrm{HCN}(1-0)}^{\prime}} \simeq 0.59 L_{\mathrm{FIR}}^{0.29}$.

The location of the new sample of LIRGs in the SFE $\mathrm{dense}_{\text {dia- }}$ grams of Figs. 2 and 3 b corroborates on a more solid statistical basis, compared to the results of Graciá-Carpio et al. (2008), that $\mathrm{SFE}_{\text {dense }}$ is on average a factor $\sim 2-3$ higher in LIRGs/ULIRGs compared to normal galaxies ( $\left\langle\mathrm{SFE}_{\text {dense }}\right\rangle$ (LIRGs/ULIRGs) $\sim 1400 \pm 100 L_{\odot} L^{\prime-1} ; \quad\left\langle\mathrm{SFE}_{\text {dense }}\right\rangle($ normal $\left.) \sim 600 \pm 70 L_{\odot} L^{\prime-1}\right)$.
This difference is a factor of 2 larger than the total uncertainty on individual data points in Fig. 2. The total change in $\mathrm{SFE}_{\text {dense }}$ expands an order of magnitude from normal galaxies to LIRGs/ULIRGs. Interestingly, two objects of the new sample, NGC 1614 and II Zw96, show the highest SFE dense values $\left(\geq 3500 L_{\odot} L^{\prime-1}\right)$ thus far reported in a galaxy. These values are close to the upper limit imposed by the maximum efficiencies measured in Galactic star-forming cores $\left(\sim 3900 L_{\odot} L^{\prime-1}\right.$; Wu et al. 2005). The resulting index derived for the $\mathrm{SFE}_{\text {dense }}$ law $(0.29 \pm 0.04)$ is higher than that obtained by Graciá-Carpio et al. (2008) (0.24 \pm 0.04$)$.

Figure 3 a shows the $L_{\mathrm{FIR}}-L_{\mathrm{HCN}(1-0)}^{\prime}$ correlation plot obtained for the full sample of galaxies. The orthogonal fit to the data points gives a superlinear correlation:

$$
\begin{aligned}
& \log L_{\mathrm{FIR}}=(1.23 \pm 0.05) \log L_{\mathrm{HCN}(1-0)}^{\prime}+(1.07 \mp 0.40) \\
& \text { or } L_{\mathrm{FIR}} \simeq 12 L_{\mathrm{HCN}(1-0)}^{\prime 1.23} .
\end{aligned}
$$

The fit is similar to that found by Graciá-Carpio et al. (2008). With the addition of the new LIRG sample we therefore confirm that the $L_{\mathrm{FIR}}-L_{\mathrm{HCN}(1-0)}^{\prime}$ correlation is significantly superlinear. This partly contradicts the first findings of Gao \& Solomon (2004a,b), who determined a power index about 1 for 


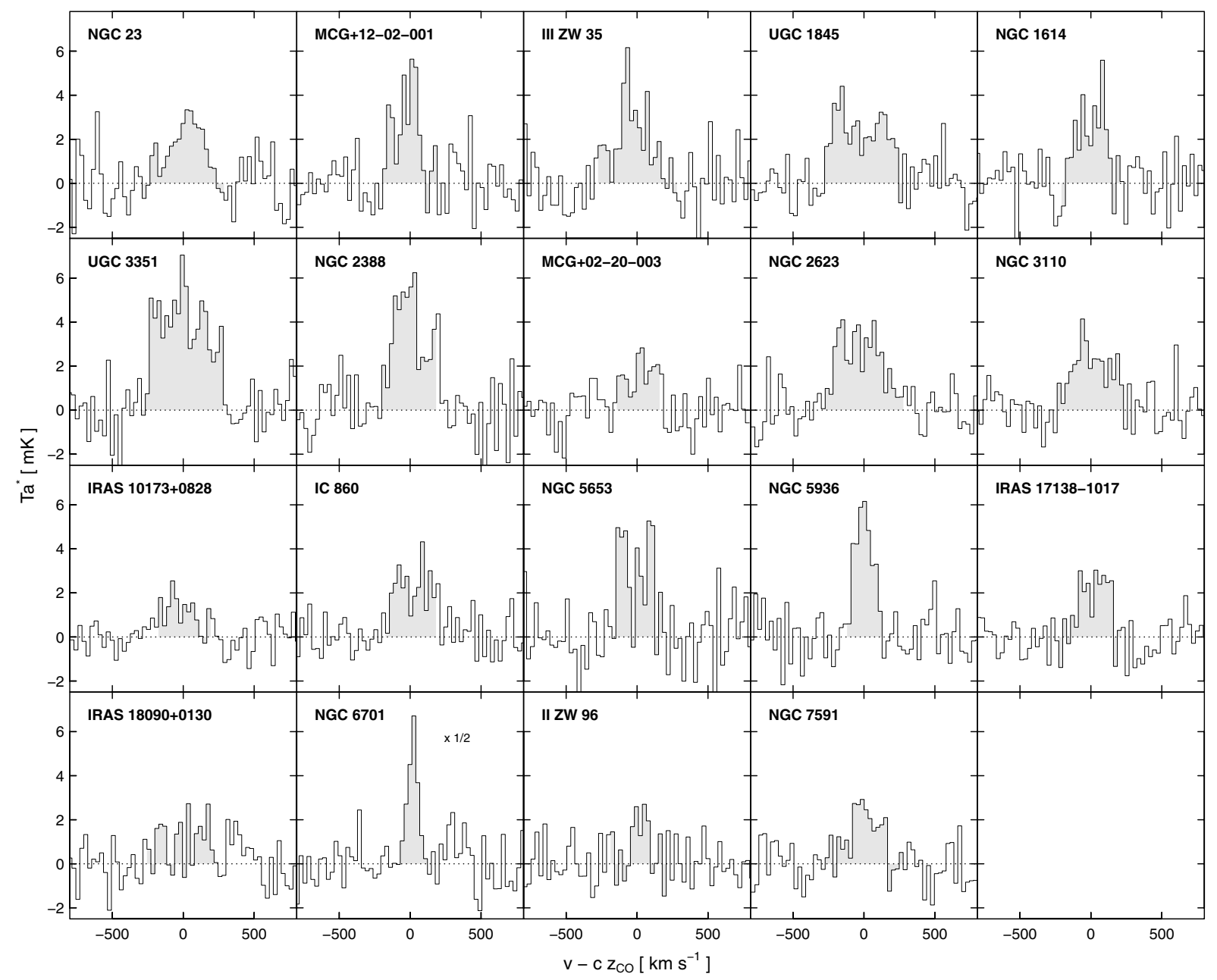

Fig. 1. b) Same as a) but showing the $\mathrm{HCN}(1-0)$ spectra.

the $L_{\mathrm{FIR}}-L_{\mathrm{HCN}(1-0)}^{\prime}$ scatter plot and a correspondingly constant $\mathrm{SFE}_{\text {dense }}$ independent of galaxy type.

Figure 4 shows the $L_{\mathrm{FIR}}-L_{\mathrm{HCO}^{+}(1-0)}^{\prime}$ correlation plot obtained for the sample of 34 LIRGs/ULIRGs for which we obtained data in the $\mathrm{HCO}^{+}$line ${ }^{1}$ (data from this work and from Graciá-Carpio et al. 2006, 2008). The orthogonal fit to the data points gives a close to linear correlation:

$$
\begin{aligned}
& \log L_{\mathrm{FIR}}=(1.06 \pm 0.15) \log L_{\mathrm{HCO}^{+}(1-0)}^{\prime}+(2.64 \mp 0.04) \\
& \text { or } L_{\mathrm{FIR}} \simeq 436 L_{\mathrm{HCO}+(1-0)}^{\prime 1.06} .
\end{aligned}
$$

We note that a similar fit using $\mathrm{HCN}(1-0)$ data but restricted to the sample of LIRGs/ULIRGs also gives a close to linear power law $(n=1.10 \pm 0.10)$. A similar fit of the $L_{\mathrm{FIR}}-L_{\mathrm{HCN}(1-0)}^{\prime}$ relation restricted to normal galaxies also gives a power index compatible with unity: $n=0.95 \pm 0.20$. Altogether this is an indication that the inclusion of both normal galaxies and LIRGs/ULIRGs in the fit of Eq. (5) is the reason that the relation between $L_{\mathrm{FIR}}$ and $L_{\mathrm{HCN}(1-0)}^{\prime}$ becomes superlinear.

Figure 5 shows the dense molecular gas depletion or exhaustion time scale $t_{\text {dep }}$ in Myr units, defined as $t_{\text {dep }} \equiv \mathrm{SFE}_{\text {dense }}^{-1} \equiv$ $M_{\text {dense }}\left[M_{\odot}\right] / S F R\left[M_{\odot} \mathrm{yr}^{-1}\right]$, as a function of $L_{\mathrm{FIR}}$. NGC 1614 and

\footnotetext{
1 There is no equivalent survey of $\mathrm{HCO}^{+}$data in a significant number of normal galaxies. The comparison between the star-formation properties of the dense molecular in LIRGs/ULIRGs and normal galaxies consequently has to rely hereafter mainly on $\mathrm{HCN}$ data.
}

II Zw96 lie at the lower end of the $t_{\text {dep }}$ distribution and are characterized by significantly short depletion time scales $\simeq 10 \mathrm{Myr}$. This is an indication that they represent extreme starburst systems that will exhaust their dense molecular gas content on time scales comparable to the typical dynamical time scales for these sources (see discussion in Sect. 7.3 and in Sect. 3.6 of AlonsoHerrero et al. 2001, for NGC 1614).

\subsection{Kennicutt-Schmidt power laws}

Figure 6 shows the KS law obtained for the different populations of galaxies compiled for this work., i.e., the star-formation rate surface density $\Sigma_{\text {SFR }}$ as a function of dense molecular gas surface density as traced by the $\mathrm{HCN}(1-0)$ line, $\Sigma_{\text {dense. }}$ In order to derive $\Sigma_{\text {SFR }}$ and $\Sigma_{\text {dense }}$ we adopted the molecular gas size estimates from published $\mathrm{CO}$ or $\mathrm{HCN}$ interferometer maps, which are available for most of the sources culled from the literature. For the new sample of LIRGs, we estimated the size of the central starforming region from the high-resolution $\mathrm{H} \alpha$ and/or $\mathrm{Pa} \alpha$ images of the galaxies published by Alonso-Herrero et al. (2006) and adopted those as representative of the typical sizes of the actively star-forming molecular gas complexes.

In Fig. 6a we represented the derived KS law. The two surface densities, $\Sigma_{\mathrm{SFR}}$ and $\Sigma_{\text {dense }}$, follow a correlation over more than 4 orders of magnitude in $\Sigma_{\text {dense }}$. An orthogonal regression 
A\&A 539, A8 (2012)

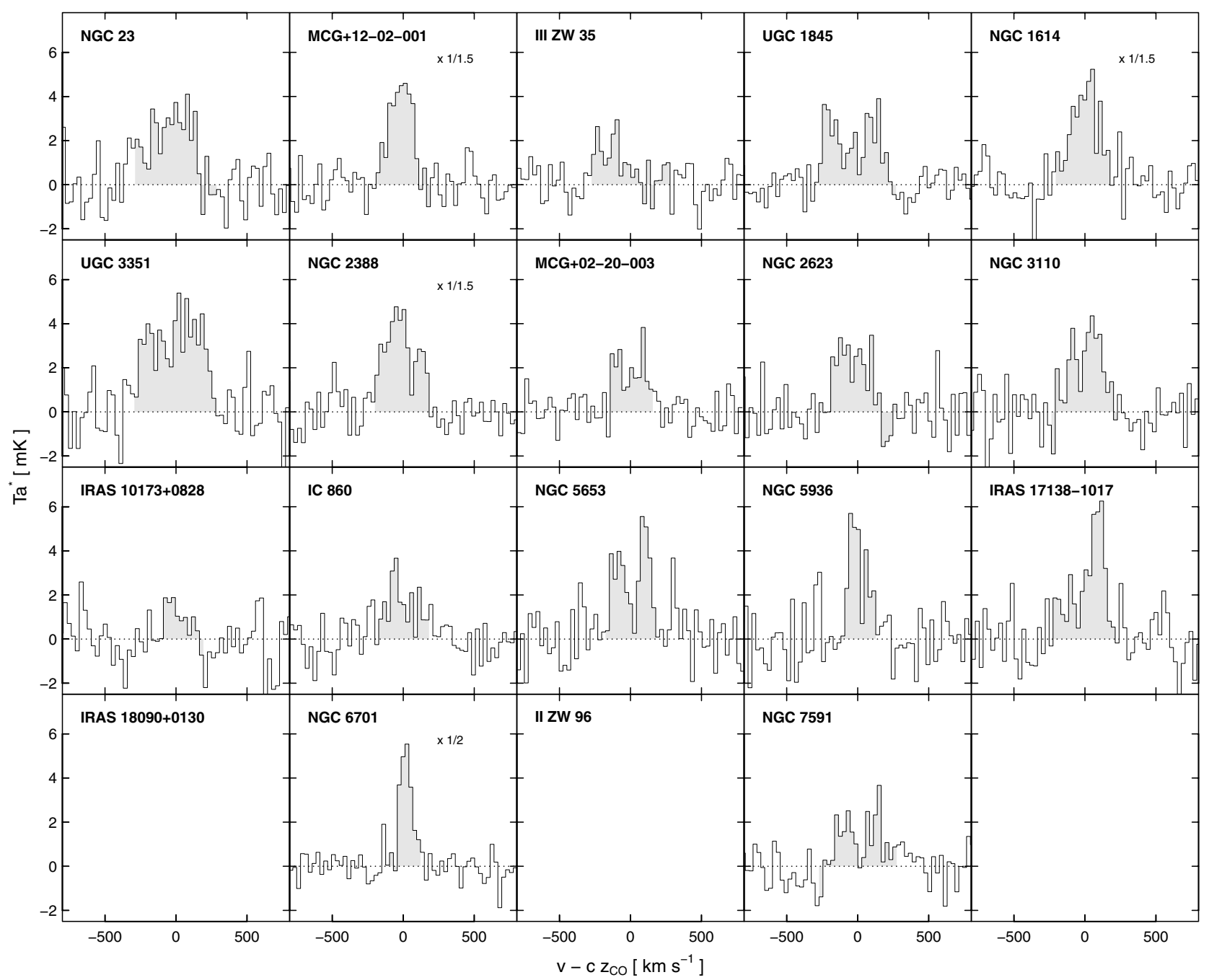

Fig. 1. c) Same as a) but showing the $\mathrm{HCO}^{+}(1-0)$ spectra.

Table 1. Main properties of the new sample of LIRGs.

\begin{tabular}{lccccccc}
\hline \hline Galaxy & $\begin{array}{c}\text { RA }_{J 2000} \\
\text { hh:mm:ss.ss }\end{array}$ & $\begin{array}{c}\text { Dec }_{\text {J2000 }} \\
\text { dd:mm:ss.s }\end{array}$ & $\begin{array}{c}d \\
\mathrm{Mpc}\end{array}$ & $z_{\mathrm{CO}}$ & $\begin{array}{c}L_{\mathrm{FIR}} \\
10^{11} L_{\odot}\end{array}$ & $\begin{array}{c}L_{\mathrm{IR}} \\
10^{11} L_{\odot}\end{array}$ & $\begin{array}{c}d_{\mathrm{SF}}{ }^{a} \\
\text { arcsec }\end{array}$ \\
\hline NGC 23 & $00: 09: 53.40$ & $+25: 55: 25.9$ & 60.1 & 0.01521 & 0.79 & 1.14 & 7.1 \\
MCG+12-02-001 & $00: 54: 04.00$ & $+73: 05: 05.5$ & 65.8 & 0.01587 & 2.04 & 2.88 & 4.7 \\
III Zw 35 & $01: 44: 30.54$ & $+17: 06: 08.8$ & 114.5 & 0.02753 & 3.79 & 4.16 & 10.0 \\
UGC 1845 & $02: 24: 07.98$ & $+47: 58: 11.0$ & 64.1 & 0.01566 & 0.95 & 1.25 & 4.3 \\
NGC 1614 & $04: 34: 00.02$ & $-08: 34: 44.9$ & 66.6 & 0.01575 & 3.04 & 4.50 & 3.1 \\
UGC 3351 & $05: 45: 47.89$ & $+58: 42: 03.9$ & 63.1 & 0.01480 & 1.52 & 1.78 & 4.5 \\
NGC 2388 & $07: 28: 53.44$ & $+33: 49: 08.7$ & 60.5 & 0.01369 & 1.38 & 1.86 & 5.2 \\
MCG+02-20-003 & $07: 35: 43.43$ & $+11: 42: 35.0$ & 73.7 & 0.01655 & 1.13 & 1.43 & 8.1 \\
NGC 2623 & $08: 38: 24.08$ & $+25: 45: 16.6$ & 82.7 & 0.01848 & 3.45 & 3.96 & 4.0 \\
NGC 3110 & $10: 04: 02.05$ & $-06: 28: 29.4$ & 77.8 & 0.01697 & 1.82 & 2.29 & 6.2 \\
IRAS 10173+0828 & $10: 20: 00.18$ & $+08: 13: 33.8$ & 220.1 & 0.04900 & 6.15 & 7.75 & 10.0 \\
IC 860 & $13: 15: 03.52$ & $+24: 37: 07.9$ & 58.9 & 0.01289 & 1.37 & 1.47 & 2.9 \\
NGC 5653 & $14: 30: 10.48$ & $+31: 12: 55.7$ & 53.4 & 0.01191 & 0.84 & 1.09 & 7.6 \\
NGC 5936 & $15: 30: 00.86$ & $+12: 59: 21.1$ & 59.0 & 0.01334 & 0.80 & 1.10 & 3.4 \\
IRAS 17138-1017 & $17: 16: 35.79$ & $-10: 20: 39.4$ & 74.2 & 0.01734 & 1.78 & 2.52 & 4.6 \\
IRAS 18090+0130 & $18: 11: 38.39$ & $+01: 31: 40.0$ & 123.5 & 0.02891 & 3.26 & 4.01 & 10.0 \\
NGC 6701 & $18: 43: 12.46$ & $+60: 39: 12.0$ & 54.5 & 0.01315 & 0.79 & 1.04 & 4.6 \\
II Zw 96 & $20: 57: 23.84$ & $+17: 07: 39.8$ & 161.0 & 0.03629 & 7.40 & 9.30 & 10.0 \\
NGC 7591 & $23: 18: 16.28$ & $+06: 35: 08.9$ & 65.4 & 0.01654 & 0.87 & 1.12 & 2.9 \\
\hline
\end{tabular}

Notes. ${ }^{(a)} d_{\mathrm{SF}}$ is the source size (major axis) of the star-forming region in arcsec determined from high-resolution optical/NIR images of hydrogen recombination lines $(\mathrm{H} \alpha, \mathrm{Pa} \alpha)$. 

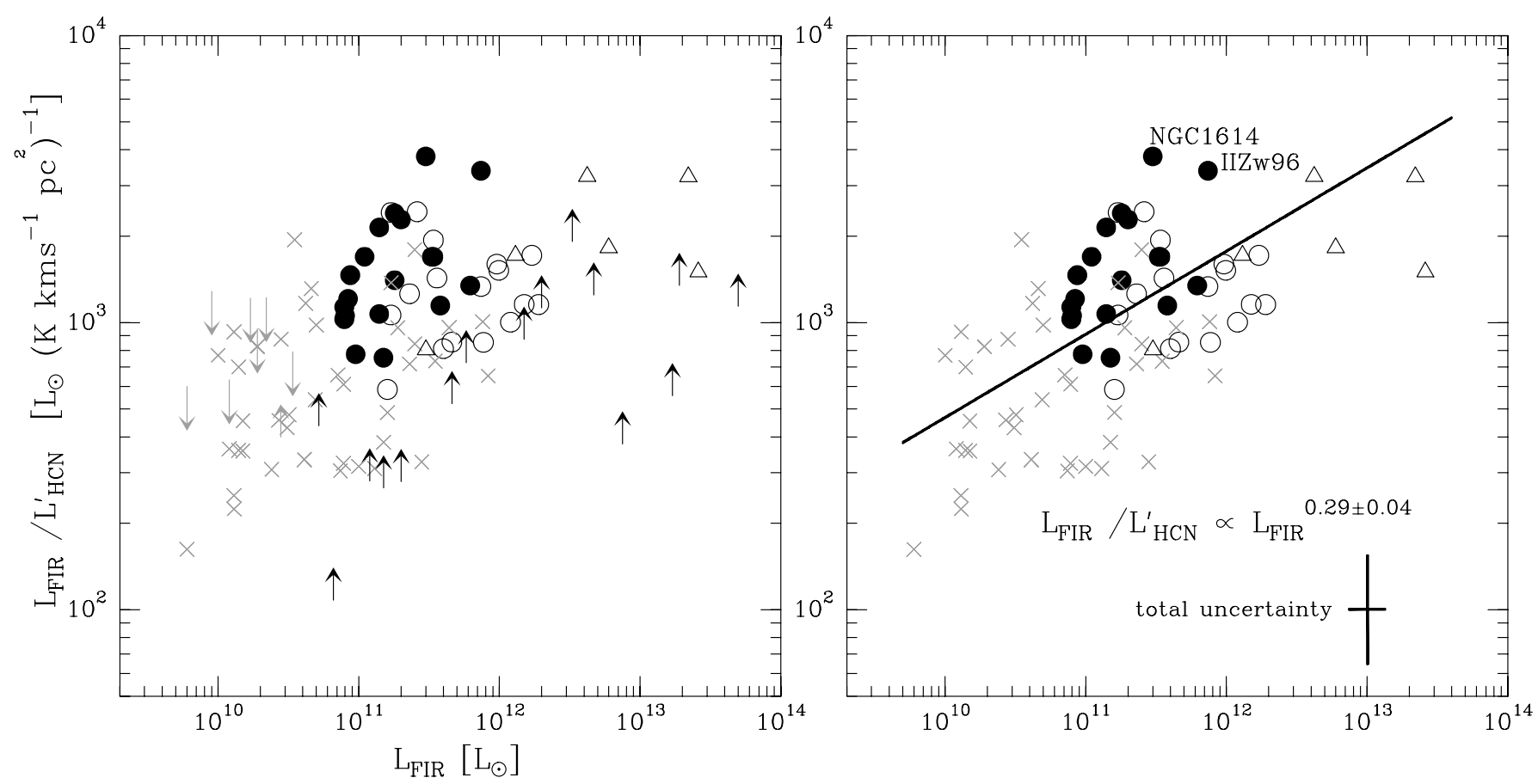

Fig. 2. a) (Left panel) We plot the $L_{\mathrm{FIR}} / L_{\mathrm{HCN}(1-0)}^{\prime}$ luminosity ratio, a proxy for the $S \mathrm{SE}_{\mathrm{dense}}$ defined in Sect. 2, as a function of $L_{\mathrm{FIR}}$ for different galaxy samples compiled in this work (see Sect. 2 for details). The different symbols represent the normal galaxies and LIRGs/ ULIRGs published by Gao \& Solomon (2004a,b; crosses), the LIRGs/ULIRGs published by Graciá-Carpio et al. (2008, empty circles), Palomar Green (PG) QSOs and high- $z$ galaxies published and compiled by Evans et al. (2006) and Gao et al. (2007), respectively (triangles), and the new sample of LIRGs published in this work (filled circles). Arrows represent upper and lower limits to $L_{\mathrm{HCN}(1-0)}^{\prime}$. b) (Right panel) Same as a), but limits are not represented. The solid line visualizes the orthogonal regression fit calculated for the full sample of objects, excluding limits. We highlight the location of NGC 1614 and II Zw96 in this plot. The total uncertainty of individual data points, including statistical and estimated systematic errors, are indicated by error bars: $\pm 0.13 \operatorname{dex}( \pm 30 \%)$ in $L_{\mathrm{FIR}}$ and $\pm 0.19 \operatorname{dex}( \pm 42 \%)$ in $L_{\mathrm{FIR}} / L_{\mathrm{HCN}(1-0)}^{\prime}$.
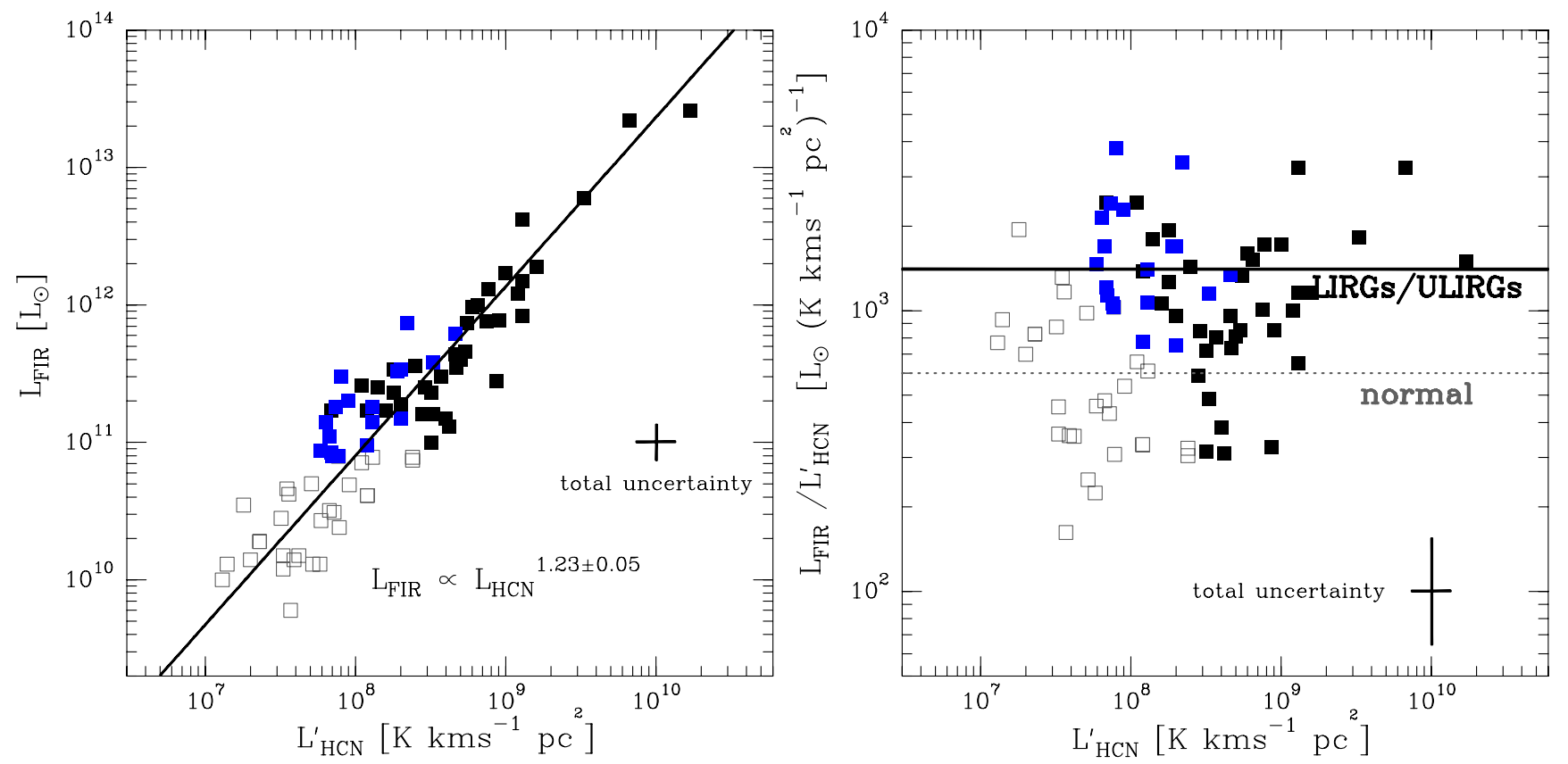

Fig. 3. a) (Left panel) $L_{\mathrm{FIR}}-L_{\mathrm{HCN}(1-0)}^{\prime}$ correlation plot derived for different galaxy samples. The solid line visualizes the orthogonal regression fit to the full sample, showing a superlinear correlation. The errorbars show the total uncertainty of individual data points $( \pm 0.13$ dex $= \pm 30 \%$ in both $L_{\mathrm{FIR}}$ and $L_{\mathrm{HCN}}^{\prime}$ ). b) (Right panel) We plot the $L_{\mathrm{FIR}} / L_{\mathrm{HCN}(1-0)}^{\prime}$ luminosity ratio as a function of $L_{\mathrm{HCN}}^{\prime}$. We display with different symbols normal galaxies ( $L_{\mathrm{IR}}<10^{11} L_{\odot}$, open squares) and luminous infrared galaxies $\left(L_{\mathrm{IR}}>10^{11} L_{\odot}\right.$, filled squares). The location of the new sample of LIRGs in this diagram is identified by blue color markers. The dashed and continuous horizontal lines indicate the average value of $L_{\mathrm{FIR}} / L_{\mathrm{HCN}(1-0)}^{\prime}$ in normal galaxies $\left(\sim 600 \pm 70 L_{\odot} L^{\prime-1}\right)$ and LIRGs/ ULIRGs $\left(\sim 1400 \pm 100 L_{\odot} L^{\prime-1}\right)$. The errorbars show the total uncertainty of individual data points: $\pm 0.13 \operatorname{dex}( \pm 30 \%)$ in $L_{\mathrm{HCN}}^{\prime}$ and $\pm 0.19 \operatorname{dex}( \pm 42 \%)$ in $L_{\mathrm{FIR}} / L_{\mathrm{HCN}(1-0)}^{\prime}$. 
Table 2. Results derived from the IRAM 30 m telescope observations.

\begin{tabular}{lrrrrrr}
\hline \hline Galaxy & \multicolumn{2}{c}{$\begin{array}{c}I_{\mathrm{CO}}{ }^{a} \\
\mathrm{~K} \mathrm{~km} \mathrm{~s}^{-1}\end{array}$} & \multicolumn{2}{c}{$\begin{array}{c}I_{\mathrm{HCN}}{ }^{a} \\
\mathrm{~K} \mathrm{~km} \mathrm{~s}^{-1}\end{array}$} & \multicolumn{2}{c}{$\begin{array}{c}I_{\mathrm{HCO}^{+}}{ }^{a} \\
\mathrm{Km} \mathrm{s}^{-1}\end{array}$} \\
\hline NGC 23 & 28.36 & $(0.32)$ & 0.81 & $(0.13)$ & 1.08 & $(0.13)$ \\
MCG+12-02-001 & 36.50 & $(0.39)$ & 0.89 & $(0.10)$ & 1.34 & $(0.10)$ \\
III Zw 35 & 10.43 & $(0.35)$ & 1.08 & $(0.15)$ & 0.50 & $(0.10)$ \\
UGC 1845 & 32.90 & $(0.31)$ & 1.29 & $(0.13)$ & 1.00 & $(0.13)$ \\
NGC 1614 & 27.16 & $(0.47)$ & 0.79 & $(0.11)$ & 1.56 & $(0.11)$ \\
UGC 3351 & 46.51 & $(0.40)$ & 2.17 & $(0.13)$ & 1.81 & $(0.13)$ \\
NGC 2388 & 42.60 & $(0.52)$ & 1.51 & $(0.11)$ & 1.69 & $(0.11)$ \\
MCG+02-20-003 & 14.08 & $(0.30)$ & 0.52 & $(0.10)$ & 0.54 & $(0.10)$ \\
NGC 2623 & 19.02 & $(0.34)$ & 1.30 & $(0.11)$ & 0.67 & $(0.12)$ \\
NGC 3110 & 31.37 & $(0.46)$ & 0.92 & $(0.10)$ & 0.87 & $(0.10)$ \\
IRAS 10173+0828 & 3.52 & $(0.26)$ & 0.43 & $(0.11)$ & 0.29 & $(0.11)$ \\
IC 860 & 9.61 & $(0.25)$ & 0.80 & $(0.10)$ & 0.55 & $(0.10)$ \\
NGC 5653 & 36.56 & $(0.57)$ & 1.00 & $(0.10)$ & 1.00 & $(0.10)$ \\
NGC 5936 & 27.44 & $(0.30)$ & 0.94 & $(0.08)$ & 0.73 & $(0.10)$ \\
IRAS 17138-1017 & 27.36 & $(0.35)$ & 0.58 & $(0.09)$ & 1.12 & $(0.13)$ \\
IRAS 18090+0130 & 19.06 & $(0.34)$ & 0.51 & $(0.11)$ & - & - \\
NGC 6701 & 36.17 & $(0.32)$ & 1.11 & $(0.17)$ & 1.15 & $(0.08)$ \\
II Zw 96 & 8.97 & $(0.32)$ & 0.35 & $(0.09)$ & - & - \\
NGC 7591 & 24.66 & $(0.31)$ & 0.61 & $(0.13)$ & 0.57 & $(0.13)$ \\
\hline
\end{tabular}

Notes. ${ }^{(a)}$ All velocity integrated intensities are in $T_{\mathrm{a}}^{*}$ scale. We list $1-\sigma$ errors within brackets.

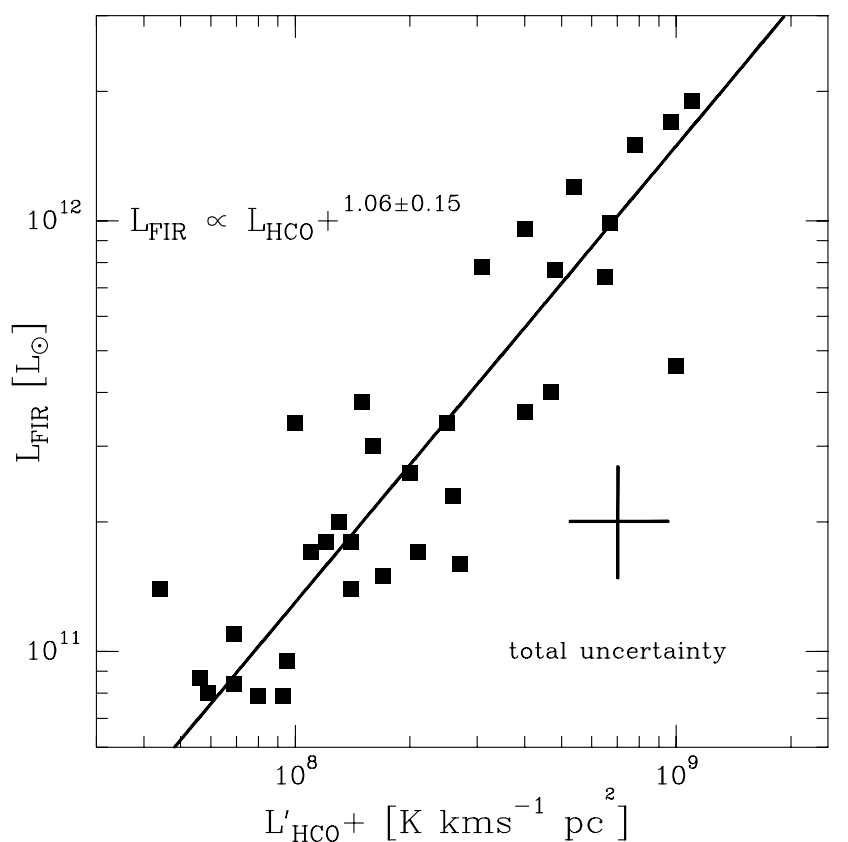

Fig. 4. $L_{\mathrm{FIR}}-L_{\mathrm{HCO}}^{\prime}(1-0)$ correlation plot derived for a subset of LIRGs/ULIRGs. The solid line visualizes the orthogonal regression fit to the sample. Errorbars as in Fig. 3.

fit to the data results in a KS-law of the dense molecular gas with a power index $N=1.12 \pm 0.04$ :

$$
\log \Sigma_{\mathrm{SFR}}=(1.12 \pm 0.04) \log \Sigma_{\text {dense }}+(-2.01 \mp 0.12)
$$$$
\text { or } \Sigma_{\mathrm{SFR}} \simeq 0.010 \Sigma_{\text {dense }}^{1.12} \text {. }
$$

The location of the new sample of LIRGs in the KS diagram of Fig. 6a helps visualize that extreme starbursts (LIRGs/ULIRGs) and normal galaxies are not fully overlapping in this scatter plot. In order to quantify if a two-function power law significantly improves the overall fit, we split the sample into normal $\left(L_{\mathrm{IR}}<10^{11} L_{\odot}\right)$ and IR luminous galaxies $\left(L_{\mathrm{IR}}>10^{11} L_{\odot}\right)$.

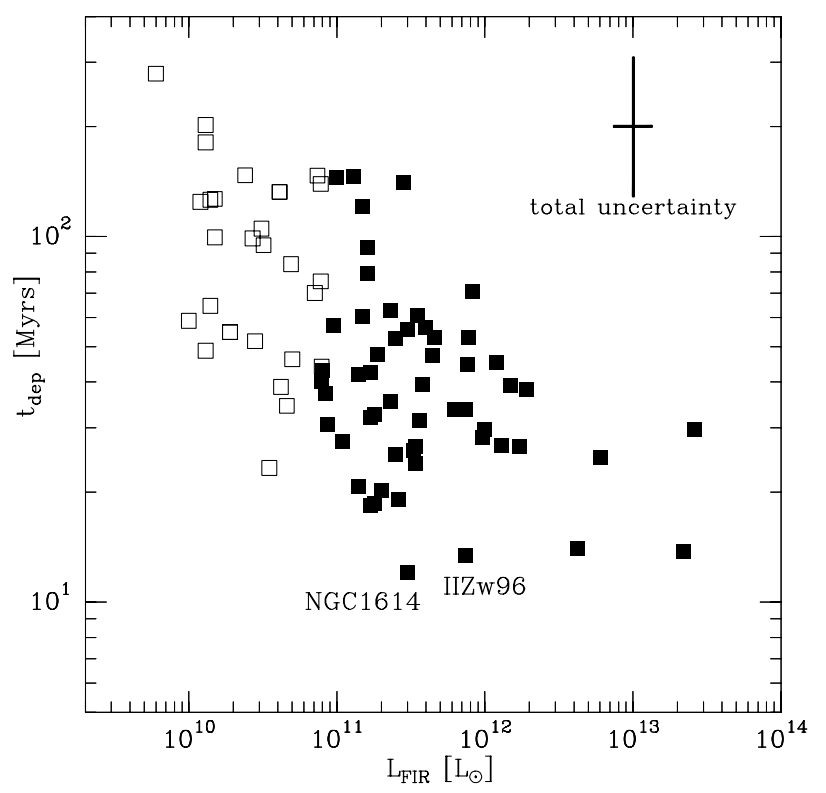

Fig. 5. Dense molecular gas depletion or exhaustion time scale, $t_{\text {dep }} \equiv 1 / \mathrm{SFE}_{\text {dense }}$ in Myr, derived for normal galaxies and LIRGs/ULIRGs. Symbols are as in Fig. 3. We highlight the location of NGC 1614 and II Zw 96 in the diagram. Errorbars show total uncertainties: $\pm 42 \%$ in $t_{\mathrm{dep}}$ and $\pm 30 \%$ in $L_{\mathrm{FIR}}$.

Figure $6 \mathrm{~b}$ shows the result of the two-function fit, which can be expressed as

$\log \Sigma_{\mathrm{SFR}}=(0.90 \pm 0.06) \log \Sigma_{\text {dense }}+(-1.71 \mp 0.15)$
or $\Sigma_{\mathrm{SFR}} \simeq 0.02 \Sigma_{\text {dense }}^{0.90}$

for galaxies with $L_{\mathrm{IR}}<10^{11} L_{\odot}$, and:

$\log \Sigma_{\mathrm{SFR}}=(1.05 \pm 0.05) \log \Sigma_{\text {dense }}+(-1.70 \mp 0.16)$

or $\Sigma_{\mathrm{SFR}} \simeq 0.02 \Sigma_{\text {dense }}^{1.05}$

for local and high- $z$ IR luminous galaxies with $L_{\mathrm{IR}} \geq 10^{11} L_{\odot}$. 
S. García-Burillo et al.: Star-formation laws in luminous infrared galaxies
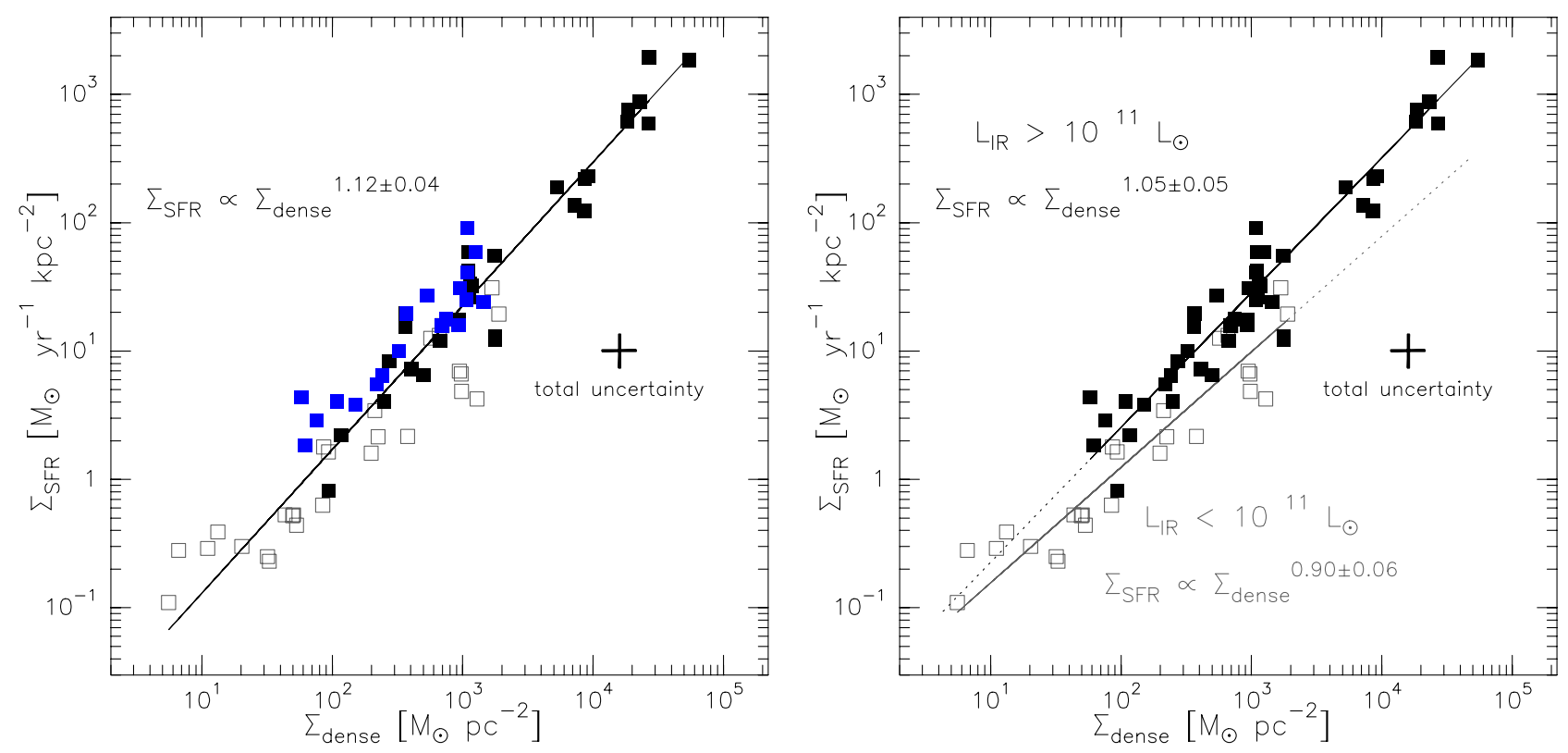

Fig. 6. a) (Left panel) Star-formation rate surface density $\Sigma_{\mathrm{SFR}}$ as a function of dense molecular gas surface density as traced by the HCN(1-0) line, $\Sigma_{\text {dense }}$, in different populations of galaxies for which we have an estimate of the molecular disk size. Symbols are as in Fig. 3. The solid black line is the orthogonal regression fit to the full sample of galaxies. b) (Right panel) Same as a), but showing the two-function power law fit to normal galaxies (gray line) and IR luminous galaxies (black line). Here, normal galaxies $\left(L_{\mathrm{IR}}<10^{11} L_{\odot}\right)$ are represented by open squares and IR luminous galaxies $\left(L_{\mathrm{IR}}>10^{11} L_{\odot}\right)$ by black filled squares as referred to in text. Errorbars show $\pm 30 \%$ uncertainties in $\Sigma_{\mathrm{SFR}}$ and $\Sigma_{\text {dense }}$.

Whereas the index of the power law is not substantially different in normal galaxies $(N=0.90 \pm 0.06)$ and LIRGs/ULIRGs $(N=1.05 \pm 0.05)$, this fit reinforces the idea of duality hinted at in the solution that Graciá-Carpio et al. (2008) found using a smaller sample of LIRGs/ULIRGs. As illustrated in Fig. 6b, the extrapolation of the KS law fitting normal galaxies to the highest values of $\Sigma_{\text {dense }}\left(\sim\right.$ a few $\left.10^{4} M_{\odot} \mathrm{pc}^{-2}\right)$ underpredicts the $\Sigma_{\mathrm{SFR}}$ in IR luminous galaxies by up to a factor $\sim 5$. This is a factor $\sim 4$ larger than the total uncertainty on $\Sigma_{\mathrm{SFR}}$. Within the range of gas surface densities share by normal galaxies and LIRGs/ULIRGs, $\Sigma_{\text {dense }} \sim 10^{2}-2 \times 10^{3} M_{\odot} \mathrm{pc}^{-2}$, the disagreement between the two laws stays within a factor 2 to 3 , i.e., still a factor $\sim 2-2.5$ larger than the total uncertainty on $\Sigma_{\mathrm{SFR}}$.

We have evaluated the goodness of the two-function power law fit by a standard $\chi^{2}$ analysis. We find that $\chi^{2}$ decreases by a factor of 1.4 in the dual fit compared to the single power law, an indication that the dual KS law for the dense gas qualifies as a better description of the data. This result is reminiscent of the bimodality found in star-formation laws derived in normal star-forming galaxies and mergers (LIRGs, ULIRGs and SMGs) from CO line data (Genzel et al. 2010; Daddi et al. 2010). We nevertheless analyze in Sect. 5 the potential biases of the twofunction fit of Eqs. (11)-(14), motivated by the division of our sample at $L_{\mathrm{IR}}=10^{11} L_{\odot}$.

In our derivation of KS laws we used different methods to derive the typical sizes assumed to be common for the actively star-forming regions and the dense molecular gas disks. This is certainly a source of uncertainty, already included in our error budget. We note, however, that a possible bias will affect the estimated $\Sigma_{\text {SFR }}$ and $\Sigma_{\text {dense }}$ to the same extent. These uncertainties would shift all data points along trajectories that correspond to straight lines of slope unity. The fits found for the KS laws are very close to the linear behavior and the described uncertainties associated to source sizes therefore cannot create a fake segregation.

\section{Statistical assessment of the two-function fit}

The results found in the previous section suggest that the KS law for the dense gas in LIRGs/ULIRGs differs from that in normal star-forming galaxies. In this section we quantify this conclusion by addressing the statistical biases inherent to these results.

\subsection{Bimodality}

We first analyze if the star-formation laws derived from $\mathrm{HCN}$ qualify as bimodal. The definition of bimodality is very strict because it requires the existence of two distinct populations within a given sample with few if any objects connecting the two populations, reflecting two different modes. We can assess if the star-formation laws derived from HCN qualify as strictly bimodal by inspecting the distribution of depletion times. Figure 7 shows histograms of $t_{\text {dep }}$ in the whole sample and in the groups of LIRGs/ULIRGs and of normal galaxies derived using different approaches of the assumed conversion factors. As expected from the difference in the mean values derived in Sect. 4, LIRGs/ULIRGs and normal galaxies tend to populate different regions of the joint histogram. This suggests a bimodal distribution with two unequally populated peaks. Assuming the non-universal conversion factors discussed in Sect. 6 certainly increases the spread in $t_{\text {dep }}$ between normal galaxies and LIRGs/ULIRGs (Fig. 7).

Hartigan \& Hartigan (1985) proposed a test that measures the goodness of unimodality as the best description of a statistical sample. The test measures the dip of a sample, defined as the maximum distance between a given empirical distribution and its corresponding best-fitting unimodal distribution. For a given sample size, the deeper the dip, the less likely it is that the distribution is unimodal. An updated version of the Hartigan dip test is implemented in the $\mathrm{R}$ package, a free software environment for 

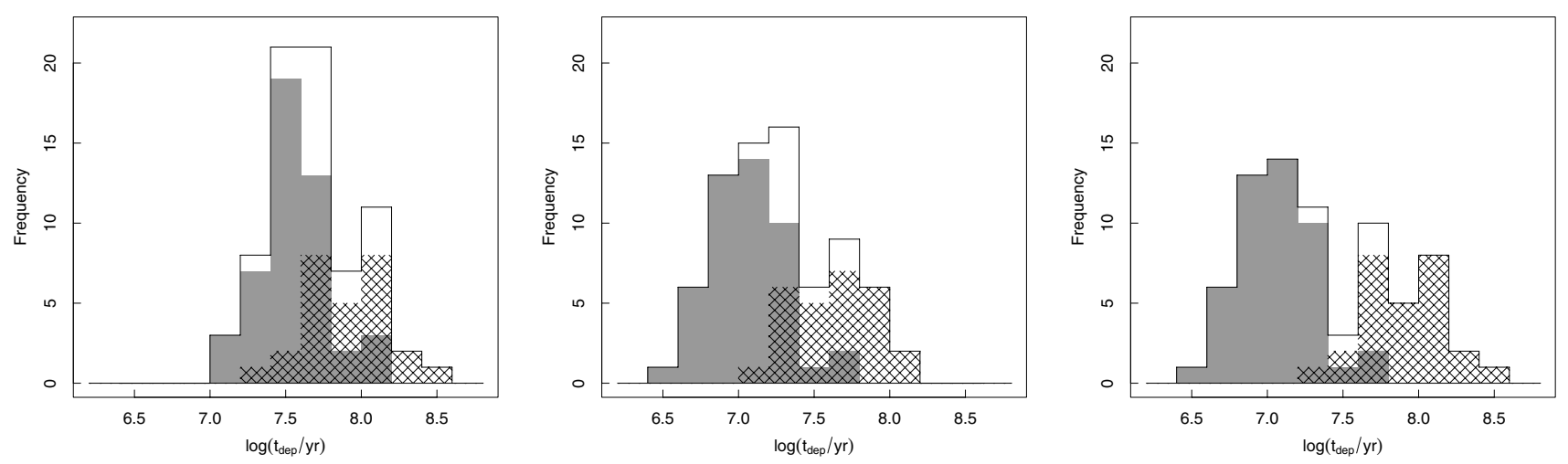

Fig. 7. Histograms of depletion times for the dense gas in the whole sample of galaxies (solid line) and in the populations of LIRGs/ULIRGs (gray filled) and of normal galaxies (hatched). The depletion times in the left-hand panel are calculated assuming the same FIR-to-SFR and HCNto-mass conversion factors for the two populations. In the middle panel, the two conversion factors are modified (see Sect. 6), whereas in the right-hand panel only the $\mathrm{HCN}$-to-mass factor is changed.

statistical computing ${ }^{2}$. We applied it to our sample of galaxies to evaluate if the set of depletion times is compatible with an unimodal distribution. The test indicates that the dip of an unimodal distribution could be as deep as measured for the depletion times in $\sim 90 \%$ of the cases, which otherwise implies that unimodality is far from being rejected. These admittedly high probabilities decrease to $\sim 80 \%$ if we assume the revised conversion factors for $\Sigma_{\text {SFR }}$ and $\Sigma_{\text {dense }}$ discussed in Sect. 6, and could reach $\sim 20 \%$ if we only changed the conversion factor for $\Sigma_{\text {dense }}$, i.e., if we adopted a similar approach to that of Genzel et al. (2010).

We conclude that we cannot claim that the star-formation process is strictly bimodal on the basis of the depletion timescale distribution. This conclusion holds particularly where universal conversion factors are adopted.

\subsection{Universality of the KS law for the dense gas}

That the two-function fit is a significant improvement over the one-function fit nevertheless suggests an underlying duality in the distribution. Because of our previous arbitrary splitting of the galaxy sample into two groups, defined by the dividing line at $L_{\mathrm{IR}}=10^{11} L_{\odot}$, the evaluation of average galaxy properties in the two subsamples is subject to potential biases, however. The underlying reason is that the selection cut used to separate the populations is not orthogonal to the variables that are being fit.

Below we test the null hypothesis that a single KS law with a fixed power law index for both LIRGs/ULIRGs and normal galaxies (e.g., Eq. (9)) is a suitable representation of our data. This hypothesis can be rejected if, on the assumption that it holds true, the measured values of three chosen observables are unlikely to result from pure random scatter. The observables used as constraints are

- The scatter of the data with respect to the one-function power law fitted to the data (Eq. (9)) $\sigma_{1 \text { fit }} \equiv \sqrt{S S R_{1 \text { fit }} /(\mathcal{N}-2)}$. $S S R_{1 \text { fit }}$ is the sum of squared residuals and $\mathcal{N}$ is the number of sources. The observable $\sigma_{1 \text { fit }}$ tells us the amount of random scatter we can plausibly assume. The value derived for $\sigma_{1 \text { fit }}$ is 0.20 dex.

- The difference, in median, between the logarithms of the depletion times of the two populations $\Delta \log \left(t_{\mathrm{dep}}\right) \equiv$ $\operatorname{median}\left\{\log \left(t_{\text {dep }}\right)\right\}_{\text {normal }}-\operatorname{median}\left\{\log \left(t_{\text {dep }}\right)\right\}_{(\mathrm{U}) \text { LIRG }}$. The measured value for $\Delta \log \left(t_{\mathrm{dep}}\right)$ is $0.45 \mathrm{dex}$. The parameter

\footnotetext{
2 http://www.r-project.org/
}

$\Delta \log \left(t_{\mathrm{dep}}\right)$ measures the difference between LIRGs/ULIRGs and normal galaxies, although it is biased toward positive values. The reason is that $\Sigma_{\mathrm{SFR}}$ is essentially proportional to $L_{\mathrm{IR}}$, so that a positive noise-driven error in the $L_{\mathrm{IR}}$ of a galaxy simultaneously underestimates its $t_{\mathrm{dep}}$ and increases the probability that the object is classified as LIRG/ULIRG. Superlinear KS laws as that of Eq. (9) (slope 1.12) enhance this bias.

- The ratio of $S S R_{2 \mathrm{fit}} / S S R_{1 \mathrm{fit}}$, where $S S R_{2 \mathrm{fit}}$ is the sum of squared residuals when using two functions to fit the data as in Sect. 4.2. The measured value for this ratio is about 0.70 . This ratio measures the improvement in the fit when separate KS laws are adopted for the two populations. $S S R_{2 \text { fit }} / S S R_{1 \text { fit }}$ is always smaller than or equal to one, since a two-function fit can always match or even improve the solution of the one-function fit.

To calculate the corresponding probability distributions of the observables, we proceeded as follows:

- We assumed that the intrinsic distribution of data in the $\left(\log \Sigma_{\text {dense }}, \log \Sigma_{\text {SFR }}\right)$ plane is given by the corrected values that result from the orthogonal least-squares fit to our observations derived in Sect. 4.2. These points follow Eq. (9) by construction.

- We evaluated the effect of the scatter of the distribution on the definition of the subsamples by scrambling our sample independently along the $\Sigma_{\text {dense }}$ and $\Sigma_{\text {SFR }}$ axes with a Gaussian scatter, hereafter denoted as $\sigma_{\text {rand }}$. The galaxies were then reclassified either as LIRG/ULIRG or as normal after rescaling the IR luminosity of each object by the randomized-toobserved ratio of $\Sigma_{\mathrm{SFR}}$. This mimics the effect of errors on the classification.

- The scatter parameter $\sigma_{\text {rand }}$ appears as a free parameter in our analysis, since there may be sources of random scatter other than the observational error $(0.13 \mathrm{dex})$. We chose 10 values of $\sigma_{\text {rand }}$ from $\sim 0$ dex to 0.9 dex and performed $5 \times 10^{3}$ randomizations for each value. Each randomized set of data was analyzed in the same way as the observed data to derive the probability distributions of our observables as a function of $\sigma_{\text {rand }}$.

The left column of Fig. 8 shows the $2.5 \%, 25 \%, 50 \%, 75 \%$ and $97.5 \%$ percentiles of $\sigma_{1 \text { fit }}$ (top), $\Delta \log \left(t_{\text {dep }}\right)$ (middle) and $S S R_{2 \text { fit }} / S S R_{1 \text { fit }}$ (bottom) as a function of $\sigma_{\text {rand }}$ assuming the null 
S. García-Burillo et al.: Star-formation laws in luminous infrared galaxies
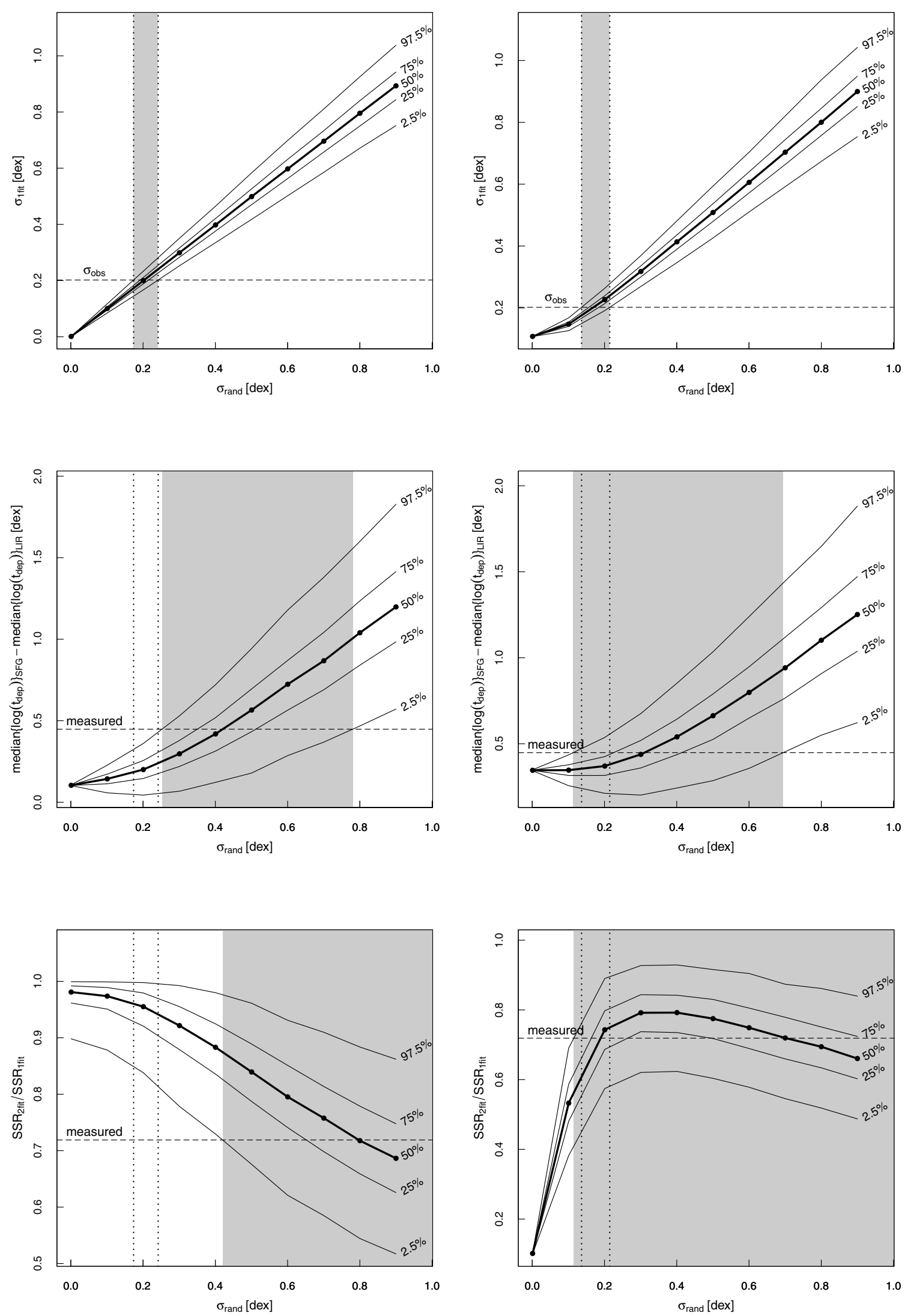

Fig. 8. Results from testing the null hypotheses of a single KS law (left column) and of a double KS law (right column). Each panel shows the $2.5 \%, 25 \%, 50 \%, 75 \%$ and $97.5 \%$ percentiles of $\sigma_{1 \text { fit }}(t o p), \Delta \log \left(t_{\mathrm{dep}}\right)$ (middle) and $S S R_{2 \mathrm{fit}} / S S R_{1 \mathrm{fit}}$ (bottom) as a function of $\sigma_{\text {rand. }}$ The measured values are indicated by dashed horizontal lines. A gray rectangle represents the $95 \%$ confidence interval for $\sigma_{\text {rand }}$ derived in each panel from the measured value. Dotted lines show the confidence intervals derived from $\sigma_{1 \text { fit }}$ (top panels). 
hypothesis. From each panel we can derive a $95 \%$ confidence interval for $\sigma_{\text {rand }}$ (gray bands in the plots) by requiring that the probability to obtain the measured value lies within the $2.5 \%-97.5 \%$ range. As shown in the top panel, $\sigma_{\text {rand }}$ should lie in the range $0.18-0.24$ dex to be compatible with our measurement of $\sigma_{1 \text { fit }}$ at the $95 \%$ confidence level. Outside that range, $\sigma_{1 \text { fit }}$ would be either lower or higher than measured ( 0.20 dex) in $97.5 \%$ of the cases. Comparing the three panels, we clearly see that this confidence interval is formally incompatible with those derived from the measured $\Delta \log \left(t_{\mathrm{dep}}\right)(0.25-0.81 \mathrm{dex})$ and $S S R_{2 \text { fit }} / S S R_{1 \text { fit }}(>0.42$ dex $)$.

We can therefore conclude that the universal one-function fit is not the best description of the data. Although the $\left(\Sigma_{\mathrm{SFR}}\right.$, $\Sigma_{\text {dense }}$ ) distribution does not qualify as strictly bimodal according to the dip test, the two-function fit to the data qualifies as a better description of the star-formation laws derived from HCN. None of the biases related to the intrinsic non-linearity of the law or to the a priori arbitrary cut in $L_{\mathrm{IR}}$ to divide the sample are able to account for the observed differences between normal galaxies and LIRGs/ULIRGs discussed in Sects. 4.1 and 4.2.

Furthermore, following a similar proof by contradiction approach, we carried out a similar test assuming a double KS law determined by the best two-function fit of Eqs. (11)-(14) discussed in Sect. 4.2. Equivalent plots to those discussed above are shown in the right column of Fig. 8. These plots clearly show that the hypothesis of a double KS law formally agrees with the measured values. Therefore, although the test cannot prove that the hypothesis of a double KS law is the only solution, we can conclude that it is a better description of the star-formation laws derived from $\mathrm{HCN}$ than a single KS law. We note that it might be possible to save the single KS law model by assuming that a significant fraction of the measured scatter is not random, but is caused by hidden variables relevant to the star-formation process such as global dynamical time scales. This alternative is explored in Sect. 7.3.

\section{Conversion factors and star-formation laws}

We discuss in Sect. 6.1 the validity of the $M_{\text {dense }}-L_{\mathrm{HCN}(1-0)}^{\prime}$ conversion factor adopted above for LIRGs/ULIRGs and consider in Sect. 6.2 the biases in estimating the SFR for normal galaxies from $L_{\mathrm{IR}}^{\mathrm{SFR}}$. The consequences of revising conversion factors for the star-formation laws obtained are discussed in Sect. 6.3.

\subsection{The HCN-to-M $M_{\text {dense }}$ conversion factor in LIRGs/ULIRGs}

It is currently accepted that the conversion factor between the CO luminosity $\left(L_{\mathrm{CO}(1-0)}^{\prime}\right)$ and the mass of molecular gas $\left(M_{\text {gas }}\right)$ is lower in mergers than in the Milky Way (MW) $\left(\alpha_{\mathrm{MW}}^{\mathrm{CO}}=\right.$ $4.8 M_{\odot} L^{\prime-1}$ ). The first conclusive evidence of a different conversion factor in mergers was found by Downes \& Solomon (1998). These authors derived that the $M_{\mathrm{gas}}-L_{\mathrm{CO}(1-0)}^{\prime}$ conversion factor in ULIRGs is $\sim 1 / 5$ of the MW's value (this lowering factor is $1 / 10$ for Arp220). More generally, the analysis of CO line emission by radiative transfer models indicates that the physical conditions found in the $\mathrm{CO}$ clouds of mergers and nuclear starbursts (densities $n\left(\mathrm{H}_{2}\right) \simeq 10^{3}-10^{4} \mathrm{~cm}^{-3}$ and brightness temperatures $T_{\mathrm{R}} \simeq 20-50 \mathrm{~K}$ ) imply that the $\mathrm{CO}$ conversion factor, which scales as $n\left(\mathrm{H}_{2}\right)^{1 / 2} / T_{\mathrm{R}}$, is typically about $(1 / 2-1 / 4) \times \alpha_{\mathrm{MW}}^{\mathrm{CO}}$ (Solomon et al. 1997; Scoville et al. 1997). A clear-cut argument in favor of a lower CO conversion factor in mergers is that the canonical MW value of $\alpha^{\mathrm{CO}}$ applied to $L_{\mathrm{CO}(1-0)}^{\prime}$ predicts gas masses that are larger than the dynamical masses in many

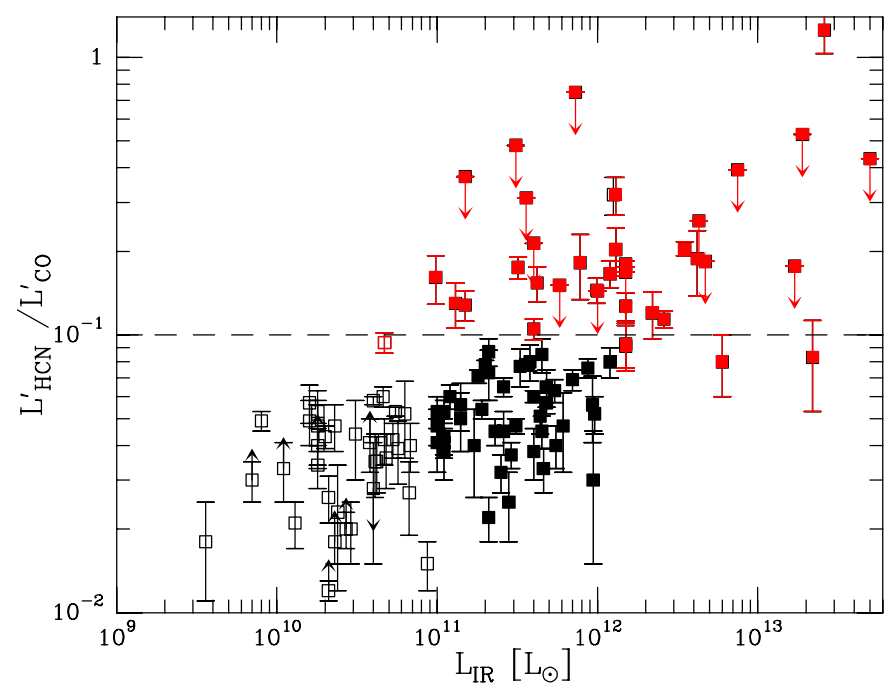

Fig. 9. HCN to CO luminosity ratios as function of $L_{\mathrm{IR}}$ for the full sample of galaxies. Here, normal galaxies $\left(L_{\mathrm{IR}}<10^{11} L_{\odot}\right)$ are represented by open squares and IR luminous galaxies $\left(L_{\mathrm{IR}}>10^{11} L_{\odot}\right)$ by filled squares. Red markers highlight those galaxies showing over-luminous $\mathrm{HCN}$ lines $(\mathrm{HCN} / \mathrm{CO} \geq 0.1)$. With the exception of one normal galaxy, all galaxies in this category are IR luminous.

LIRGs/ULIRGs at different redshift ranges (Tacconi et al. 2008; Daddi et al. 2010; Genzel et al. 2010)

There have also been claims in the literature that the MW value of the $M_{\text {dense }}-L_{\mathrm{HCN}(1-0)}^{\prime}$ conversion factor, initially adopted in Sect. 4, does not apply to mergers either (Gao \& Solomon 2004a; Graciá-Carpio et al. 2008). The high HCN to CO ratios measured in some ULIRGs/LIRGs $(\simeq 0.1)$ make the requirement that $\alpha_{\text {mergers }}^{\mathrm{CO}} \leq(1 / 2-1 / 4) \times \alpha_{\mathrm{MW}}^{\mathrm{CO}}$ mostly incompatible with the basic prescription that $M_{\text {dense }}$ should not in all likelihood exceed $0.5 \times M_{\text {gas }}($ Gao \& Solomon 2004a).

The case of the ULIRG Arp 220 is paradigmatic in this respect. Downes \& Solomon (1998) derived from the lower conversion factor for $\mathrm{CO}$ a total gas mass for the system $M_{\text {gas }} \sim 5 \times 10^{9} M_{\odot}$. The mass of dense gas derived from our HCN observations if we use the standard conversion version factor for $\mathrm{HCN}\left(\alpha_{\mathrm{MW}}^{\mathrm{HCN}}\right)$ is $M_{\text {dense }} \sim 1.2 \times 10^{10} M_{\odot}$, i.e., $\sim(2-3) \times M_{\text {gas }}$. This result indicates that $\alpha_{\mathrm{Arp} 220}^{\mathrm{HCN}}$ should be $\leq(1 / 2-1 / 3) \alpha_{\mathrm{MW}}^{\mathrm{HCN}}$ even in the extreme limit case where $M_{\text {dense }}=M_{\text {gas }}$. The new data obtained in our work for other IR luminous galaxies corroborates this picture. Figure 9 shows the $\mathrm{HCN}$ to $\mathrm{CO}$ ratios as a function of $L_{\mathrm{IR}}$ derived for the full sample of galaxies used in this paper. This diagram is used to single out overluminous $\mathrm{HCN}$ line targets in our sample, characterized by $\mathrm{HCN} / \mathrm{CO} \geq 0.1$ within the errors. About $50 \%$ of the LIRGs/ULIRGs in our sample are overluminous $\mathrm{HCN}$ galaxies, identified by the red filled square symbols in Fig. 9. By contrast, only one normal galaxy in our sample is overluminous in HCN. In these galaxies $\alpha^{\mathrm{HCN}}$ has to be lowered by a similar factor as $\alpha_{\text {mergers }}^{\mathrm{CO}}$ relative to the MW canonical values.

Following a similar approach to that employed to constrain the value of $\alpha_{\text {mergers }}^{\mathrm{CO}}$, Graciá-Carpio et al. (2008) used the data obtained in a multiline survey of $\mathrm{HCN}$ and $\mathrm{HCO}^{+}$of 17 LIRGs and ULIRGs to fit $\alpha^{\mathrm{HCN}}$ with large velocity gradient (LVG) radiative transfer codes. The main outcome of Graciá-Carpio et al.'s study also backs up the claim that $\alpha^{\mathrm{HCN}}$ is $\sim 3$ times lower at high $L_{\mathrm{FIR}}$. Lower conversion factor for $\mathrm{HCN}$ in extreme starbursts can be related to the particular chemical environment of the dense molecular gas in these sources where a hot-core like type 
S. García-Burillo et al.: Star-formation laws in luminous infrared galaxies
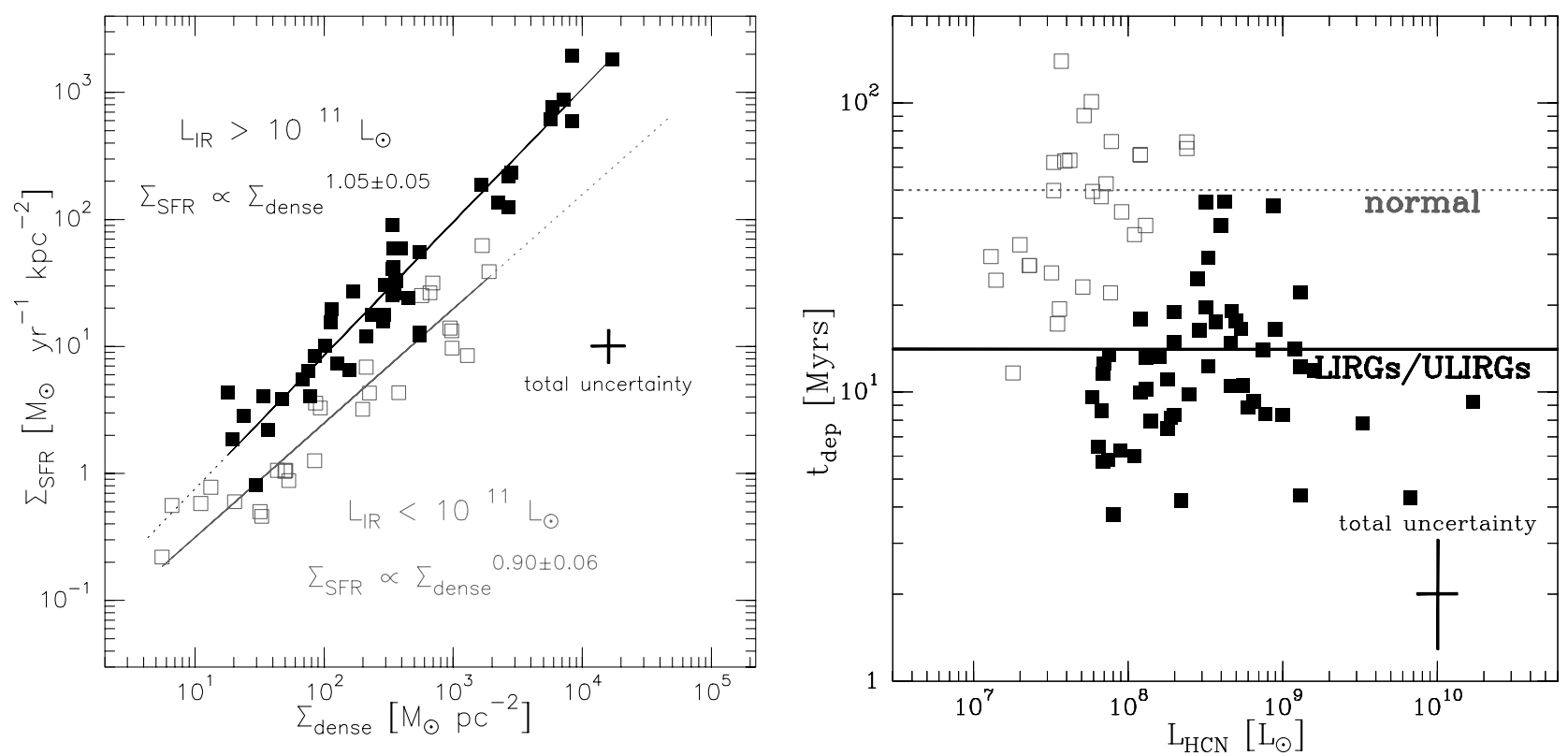

Fig. 10. a) (Left panel) Same as Fig. 6a, but showing the revised two-function power law fit to normal galaxies (gray line) and luminous infrared galaxies (black line) discussed in Sect. 6.3. Symbols and errorbars as in Fig. 6. b) (Right panel) We show the revised depletion time scale as a function of $L_{\mathrm{HCN}}^{\prime}$ derived in normal and luminous infrared galaxies, as discussed in Sect. 6.3. The dashed and continuous horizontal lines indicate the average value of the depletion time scale in normal galaxies $\left(t_{\mathrm{dep}} \sim 50 \pm 5 \mathrm{Myr}\right)$ and LIRGs/ULIRGs $\left(t_{\mathrm{dep}} \sim 14 \pm 1.4 \mathrm{Myr}\right)$. Symbols and errorbars as in Fig. 5.

of chemistry may prevail and enhance HCN abundances (e.g., Lintott et al. 2005). In addition, non-collisional excitation could also be responsible for the surprising strength of $\mathrm{HCN}$ lines in some IR luminous systems (e.g.; Aalto et al. 1995; GarcíaBurillo et al. 2006; Guélin et al. 2007; Weiß et al. 2007). In either case, the HCN conversion factor should be lowered.

The independent lines of evidence mentioned above indicate that for a sizeable fraction of the IR luminous galaxies in our sample, the HCN conversion factor should be lowered. It remains to be proved that a similar correction should be applied to all the galaxies in our sample that qualify as IR luminous mergers. With this caveat in mind we rederive the KS laws in Sect. 6.3 adopting $\alpha^{\mathrm{HCN}}=1 / 3.2 \times \alpha_{\mathrm{MW}}^{\mathrm{HCN}}$ in LIRGs/ULIRGs. This is the same rescaling factor generally adopted for mergers in the CO study of Genzel et al. (2010). We note that about $70 \%$ of the LIRGs/ULIRGs analyzed in our paper have $L_{\mathrm{IR}}>10^{11.5} L_{\odot}$, the limit beyond which mergers and strongly interacting systems start to dominate over less disturbed disk systems (Sanders \& Ishida 2004; Alonso-Herrero et al. 2009).

\subsection{The $L_{F I R}$-to-SFR conversion factor in normal galaxies}

In Sect. 4 we used the IR luminosity of the galaxies as a proxy for their SFR. While this approach is valid for dusty galaxies, it has now become clear that in normal star-forming galaxies one needs to account for both the obscured (traced by the IR luminosity) and the unobscured SFRs (Pérez-González et al. 2006; Calzetti et al. 2007; Kennicutt et al. 2009). In particular, combinations of an IR tracer (e.g., the $24 \mu \mathrm{m}$ luminosity) with the observed $\mathrm{H}_{\alpha}$ luminosities are shown to work well. In this section we evaluate the corrections needed to account for all the SFR (obscured+unobscured) in our sample of galaxies.

For the normal star-forming galaxies in our sample we used the SINGS sample data. We have eight galaxies in common with the SINGS survey. We used the integrated observed (that is, not corrected for extinction) $\mathrm{H} \alpha$ and $24 \mu \mathrm{m}$ luminosities given by Kennicutt et al. (2009) to compute the total SFR using the prescriptions given in their paper. As described in Kennicutt et al. (2009), the use of total IR or monochromatic IR luminosities work equally well to derive the total (obscured+unobscured) SFR. On average we find that $\operatorname{SFR}(24 \mu \mathrm{m}) / \operatorname{SFR}($ tot $)=0.5-0.6$. This indicates that SFR based on an IR tracer underestimates the true SFR roughly by a factor of 2 in normal star-forming galaxies (see also Kennicutt et al. 2009, and references therein).

In LIRGs and ULIRGs, as mentioned above, the IR-based SFR indicators account for most of the total SFR. This was shown by recent integral field spectroscopy studies of a sample of more than 50 LIRGs and ULIRGs (García-Marín et al. 2009; Rodríguez-Zaurín et al. 2011). Therefore no corrections were applied to derive the SFR surface density for LIRGs and ULIRGs.

We therefore adopt in Sect. 6.3 a correction factor of 2 to derive $\Sigma_{\mathrm{SFR}}$ from $L_{\mathrm{IR}}^{\mathrm{SFR}}$ in normal star-forming galaxies. This is likely an upper limit to the true correction factor in normal galaxies, because we expect that the attenuation will vary more continuously as a function of the FIR luminosity. We note, however, that applying a lower correction factor for normal galaxies would further reinforce the dual behavior of star-formation laws discussed in Sect. 6.3 (see Fig. 7).

\subsection{Revised star-formation laws}

We have fitted a new two-function power law to the whole sample of galaxies using the revised values of $\Sigma_{\text {dense }}$ (in LIRGs/ULIRGs) and $\Sigma_{\mathrm{SFR}}$ (in normal galaxies) derived according to Sects. 6.1 and 6.2. Figure 10a visualizes the new star-formation relation in normal galaxies and LIRGs/ULIRGs. The best orthogonal fit solution can be expressed as

$\log \Sigma_{\mathrm{SFR}}=(0.90 \pm 0.06) \log \Sigma_{\text {dense }}+(-1.40 \mp 0.15)$
or $\Sigma_{\mathrm{SFR}} \simeq 0.04 \Sigma_{\text {dense }}^{0.90}$ 
for galaxies with $L_{\mathrm{IR}}<10^{11} L_{\odot}$, and

$\log \Sigma_{\mathrm{SFR}}=(1.05 \pm 0.05) \log \Sigma_{\text {dense }}+(-1.17 \mp 0.16)$

or $\Sigma_{\mathrm{SFR}} \simeq 0.07 \Sigma_{\text {dense }}^{1.05}$

for local and high- $z$ IR luminous galaxies with $L_{\mathrm{IR}} \geq 10^{11} L_{\odot}$.

An inspection of Fig. 10a indicates that compared to Fig. 6b, the dual behavior is significantly reinforced in the new version of the KS laws. At the high end of $\Sigma_{\text {dense }}$ values $(\sim$ a few $10^{4} M_{\odot} \mathrm{pc}^{-2}$ ) the normal galaxy law underpredicts $\Sigma_{\mathrm{SFR}}$ in IR luminous galaxies by about an order of magnitude, a factor $\sim 8$ larger than the typical uncertainty on $\Sigma_{\mathrm{SFR}}$. Furthermore, within the range of gas surface densities shared by normal galaxies and LIRGs/ULIRGs, $\Sigma_{\text {dense }} \sim 2 \times 10^{1}-2 \times 10^{3} M_{\odot} \mathrm{pc}^{-2}$, the factor 3 to 5 disagreement between the two laws is statistically significant, being a factor $\sim 3-4$ larger than the typical uncertainty on $\Sigma_{\mathrm{SFR}}$. Instead, if we try to fit all the observations shown in Fig. 10a with a one-parameter law over the whole range of IR luminosities, we obtain

$\log \Sigma_{\text {SFR }}=(1.15 \pm 0.04) \log \Sigma_{\text {dense }}+(-1.60 \mp 0.06)$.

This solution has a 2.2 times higher $\chi^{2}$ than the two-function fit found above.

Figure $10 \mathrm{~b}$ shows the revised depletion time scales of the dense molecular gas in normal galaxies and LIRGs/ULIRGs. A similar reinforcement of the duality in star-formation laws can be identified if we compare the new estimates of $t_{\text {dep }}$ with those shown in Fig. 5. The value of $t_{\text {dep }}$ is on average a factor $\sim 3-$ 4 lower in LIRGs/ULIRGs $\left(t_{\text {dep }} \sim 14 \pm 1.4 \mathrm{Myr}\right.$ ) compared to normal galaxies $\left(t_{\mathrm{dep}} \sim 50 \pm 5 \mathrm{Myr}\right)$. This difference is a factor of 2-3 larger than the total uncertainty of individual data points.

As extensively discussed in Sect. 5, the revised starformation laws of Eqs. (15)-(18), by increasing the spread between normal galaxies and LIRGs/ULIRGs, make the twofunction fit as the best description of the data more plausible.

\section{Star-formation models: local vs. global}

The evidence of bimodality in star-formation laws derived from CO line data is a challenge for star-formation models relying only on local processes (e.g., Genzel et al. 2010). Our findings of a similar yet admittedly less extreme duality in the starformation laws derived from $\mathrm{HCN}$, a better tracer of the dense molecular phase, and thus more directly related to star formation, can put stringent constraints on models. In contrast with the highly scattered CO diagram, the lower intrinsic dispersion in the HCN star-formation laws helps assess the verisimilitude of duality with a higher statistical significance. We discuss in Sect. 7.1 to what extent our results based on the HCN observations can be used to test one of the key predictions of the starformation model of Krumholz \& McKee (2005): the constancy of the star-formation rate per free-fall time $\left(\mathrm{SFR}_{\mathrm{ff}}\right)$. We study in Sect. 7.2 the values predicted by Krumholz et al.'s models for the $L_{\mathrm{FIR}} / L_{\mathrm{HCN}(1-0)}^{\prime}$ luminosity ratios in different galaxy populations and use our data to benchmark the models. Furthermore, we study in Sect. 7.3 whether the inclusion of "global" dynamical time scales offers a better description of the star-formation laws derived for the dense gas.

\subsection{Observations vs. models: is the star-formation rate per free-fall time constant?}

Krumholz \&McKee (2005) developed an analytic prescription for the star-formation laws that are valid for different galactic environments. In their model star formation is globally very inefficient because it only takes place in a small subregion of a supersonic turbulent virialized molecular cloud. In this overdense subregion, known as the cloud core(s), gravitational pull is able to take over kinetic energy and the collapse starts. If SFR is the total star-formation rate in a galaxy, the star-formation rate per free-fall time in objects of class $\mathrm{X}, \mathrm{SFR}_{\mathrm{ff}-\mathrm{X}}$, can be obtained as a function of SFR and the total mass of X objects, $M_{\mathrm{X}}$, (or of their associated variables SFE or $t_{\mathrm{dep}}$ ), as well as of the fraction of star formation occurring in these objects, $f_{\mathrm{X}}$ and the free-fall time, $t_{\mathrm{ff}-\mathrm{X}}$ :

$\mathrm{SFR}_{\mathrm{ff}-\mathrm{X}}=\frac{f_{\mathrm{X}} \mathrm{SFR} t_{\mathrm{ff}-\mathrm{X}}}{M_{\mathrm{X}}}=f_{\mathrm{X}} \mathrm{SFE}_{\mathrm{X}} t_{\mathrm{ff}-\mathrm{X}}=f_{\mathrm{X}} \frac{t_{\mathrm{ff}-\mathrm{X}}}{t_{\mathrm{depl}}}$.

The star-formation rate per free-fall time is a function of two parameters: the ratio of the kinetic energy (thermal plus turbulent) to the gravitational energy of the cloud $\left(\alpha_{\mathrm{VIR}}\right)$ and the Mach number of the region $(\mathcal{M})$ where $\mathcal{M}^{2}$ is roughly the ratio of the kinetic energy to the thermal energy. One of the key predictions of these models is that the dimensionless SFR $_{\mathrm{ff}}$ is expected to be low and lie within a quite restricted range $\leq 3 \%(\sim 0.011-0.028)$ for the entire span of plausible values of $\alpha_{\mathrm{VIR}}(\sim 1-2)$ and $\mathcal{M}$ $(\sim 20-80)$. This offers a quantitative description for why star formation is so inefficient in a highly turbulent molecular medium.

If we take $\mathrm{HCN}$ clouds as representative objects, then $\mathrm{SFE}_{\mathrm{X}} \equiv \mathrm{SFE}_{\mathrm{dense}} \equiv 1 / t_{\mathrm{dep}}$ as defined in Sect. 4.1. We can therefore estimate $\mathrm{SFR}_{\mathrm{ff}}$ using Eq. (20) directly from observations, provided that we assume a typical density for $\mathrm{HCN}$ clouds and suppose a value for $f_{\mathrm{X}}$. It is reasonable to assume that $f_{\mathrm{X}}$ is about unity because all star formation in galaxies is likely taking place at densities exceeding the critical densities of the HCN $(1-$ $0)$ line $\left(n_{\mathrm{HCN}}^{\text {crit }}\left(\mathrm{H}_{2}\right) \sim 3 \times 10^{4} \mathrm{~cm}^{-3} / \tau\right.$, where $\tau$ is the opacity of the 1-0 line of HCN; e.g., Gao \& Solomon 2004a). The actual value of $t_{\mathrm{ff}-\mathrm{HCN}}$ depends on the density of clouds emitting HCN. All in all, observations can be used to verify/falsify the prediction of Krumholz \& McKee (2005)'s model about the behavior of $\mathrm{SFR}_{\mathrm{ff}}$.

Krumholz \& Tan (2007) used galactic and extragalactic observations of a variety of objects to derive $\mathrm{SFR}_{\mathrm{ff}-\mathrm{X}}$ as a function of the characteristic ISM densities, which are probed by different molecular tracers (i.e., X-objects) in their compilation. A key outcome of their work is that $\mathrm{SFR}_{\mathrm{ff}-\mathrm{X}}$ seems to stay roughly constant with density. However, the results obtained from HCN suggest a significant deviation of $\mathrm{SFR}_{\mathrm{ff}-\mathrm{X}}$ from this general trend. The average value of $\mathrm{SFR}_{\mathrm{ff}}$ derived from the HCN observations used in their paper $\left(\mathrm{SFR}_{\mathrm{ff}-\mathrm{HCN}} \sim 0.0058\right)$ lies below the range predicted by Krumholz \& McKee (2005)'s model. Krumholz \& Tan (2007) nevertheless assigned a large error bar to $\mathrm{SFR}_{\mathrm{ff}-\mathrm{HCN}}$, implicitly assuming that the scattered distribution of $\mathrm{SFR}_{\mathrm{ff}}$ derived from $\mathrm{HCN}$ data is mostly random ${ }^{3}$.

We use below the HCN data discussed in this work to study the distribution of $\mathrm{SFR}_{\mathrm{ff}}$ values derived in our sample of galaxies. Our goal is to check if the new observations are compatible with the prediction of a constant $\mathrm{SFR}_{\mathrm{ff}}$. In this comparison we use the two versions of the star-formation laws derived using the standard conversion factors (Case-I below) and the revised conversion factors discussed in Sect. 6 (Case-II below). As discussed below, exploring different conversion factors is paramount if we are to probe the plausible range of values for $\mathrm{SFR}_{\mathrm{ff}}$, because these two quantities are interconnected through Eq. (20).

\footnotetext{
3 Krumholz \& Tan (2007) claim that uncertainties in observations translate into an order of magnitude errorbar.
} 

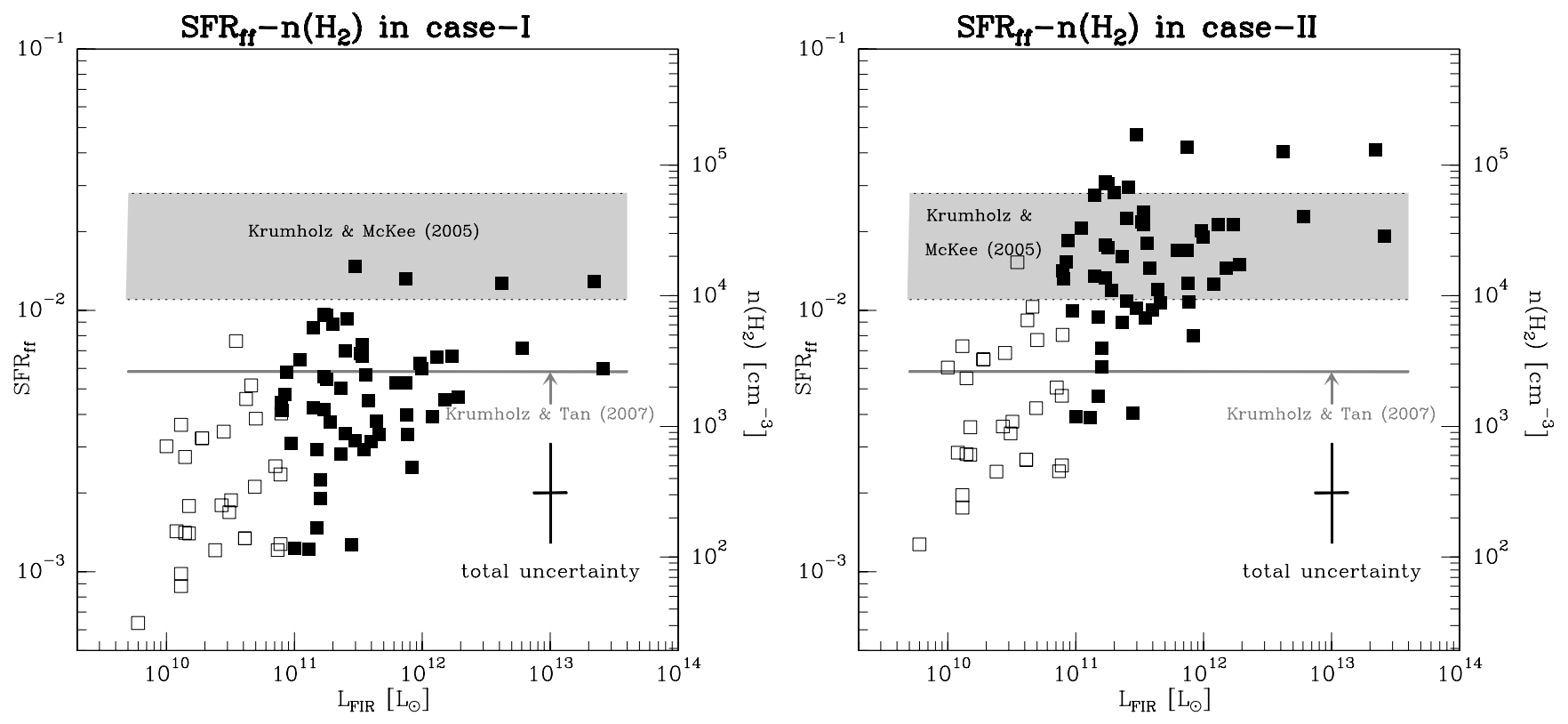

Fig. 11. a) (Left panel) Star-formation rate per free-fall time $\left(\mathrm{SFR}_{\mathrm{ff}}\right.$; shown in the left $Y$ axis) derived from the observed $\mathrm{SFE}_{\text {dense }}$ in different populations of galaxies assuming a characteristic gas density for the $\mathrm{HCN}$ cloud $n_{\mathrm{HCN}}\left(\mathrm{H}_{2}\right)=3 \times 10^{4} \mathrm{~cm}^{-3}$. We show on the right $Y$ axis the value of $n_{\mathrm{HCN}}\left(\mathrm{H}_{2}\right)$ derived from $\mathrm{SFE}_{\text {dense }}$ assuming a constant $\mathrm{SFR}_{\mathrm{ff}}=0.02$. This value lies within the most likely range for SFR $\mathrm{ff}_{\mathrm{ff}} \sim 0.011-0.028$ as determined by the star-formation model of Krumholz \& McKee (2005). We also highlight the average value of SFR $\mathrm{ff}_{\mathrm{ff}} \sim 0.0058$ as determined by Krumholz \& Tan (2007) from a compilation of galactic and extragalactic HCN observations. Symbols are as in Fig. 3. Errorbars on SFR $\mathrm{ff}( \pm 42 \%)$ and $L_{\mathrm{FIR}}( \pm 30 \%)$ are shown. b) (Right panel) Same as a) but obtained based on the revised values of $\Sigma_{\text {dense }}$ (in LIRGs/ULIRGs) and $\Sigma_{\text {SFR }}$ (in normal galaxies) discussed in Sect. 6.

\subsubsection{Case-l: standard conversion factors}

If we assume that all $\mathrm{HCN}$ clouds have densities equal to $n_{\mathrm{HCN}}^{\text {crit }}=3 \times 10^{4} \mathrm{~cm}^{-3}$ (i.e., we take $\tau=1$ ) we can obtain $\mathrm{SFR}_{\mathrm{ff}}$ from Eq. (20). Figure 11a represents $S F R_{\mathrm{ff}}$ as a function of $L_{\mathrm{FIR}}$ obtained from the $\mathrm{SFE}_{\text {dense }}$ values derived in Sect. 4, i.e., prior to the correction of conversion factors discussed in Sect. 6. We note that the distribution of SFR $\mathrm{ff}$ lies noticeably below the range predicted by the model, as shown in Fig. 11a. In addition to this conspicuous downward shift, the deduced $\mathrm{SFR}_{\mathrm{ff}}$ distribution shows a systematic trend with $L_{\text {FIR }}$ and thus cannot be described as random. The order of magnitude increase in $S_{F} R_{\mathrm{ff}}$ from normal galaxies to LIRGs/ULIRGs is statistically significant and it is about a factor of 7 higher than the typical uncertainty of individual data points $(42 \%)$. The $\mathrm{SFR}_{\mathrm{ff}}$ progression echoes the $\mathrm{SFE}_{\text {dense }}$ trend with $L_{\mathrm{FIR}}$ discussed in Sect. 4.1.

To render a constant $\mathrm{SFR}_{\mathrm{ff}}$ compatible with the order of magnitude increase in $\mathrm{SFE}_{\text {dense, }}$, the density of $\mathrm{HCN}$ clouds has to change notably from normal galaxies to LIRGs/ULIRGs, as $n_{\mathrm{HCN}}\left(\mathrm{H}_{2}\right)$ scales as $\sim \mathrm{SFE}_{\text {dense }}^{2}$. We represent in the right $Y$ axis of Fig. 11a the value of $n_{\mathrm{HCN}}\left(\mathrm{H}_{2}\right)$ required to fit observations, assuming a fixed $\mathrm{SFR}_{\mathrm{ff}}=0.02$ for all galaxies, which is close to the average value predicted by the star-formation model of Krumholz \& McKee (2005). The required $n_{\mathrm{HCN}}\left(\mathrm{H}_{2}\right)$ densities span two orders of magnitude from normal galaxies $\left(\sim 10^{2} \mathrm{~cm}^{-3}\right)$ to LIRGs/ULIRGs $\left(\sim 10^{4} \mathrm{~cm}^{-3}\right)$. These densities are exceedingly low compared to $n_{\mathrm{HCN}}^{\text {crit }}$; this poses a problem for the standard scenario of collisional excitation of $\mathrm{HCN}$ lines. Extreme and therefore unrealistic high opacities for $\mathrm{HCN}$ would be required to compensate for the low densities: $\tau_{\mathrm{HCN}}=3-300$. As an additional relevant constraint, we would need to assume that opacities are a factor 10-100 higher in normal galaxies compared to LIRGs/ULIRGs. This requirement is at odds with the commonly measured higher molecular abundances of extreme starbursts (Combes 1991; Wild et al. 1992; Nguyen et al. 1992; Krips et al. 2008; Graciá-Carpio et al. 2008). The current observational evidence contradicts the existence of these abnormally low HCN densities, at least in the galaxies for which reliable estimates of $n_{\mathrm{HCN}}\left(\mathrm{H}_{2}\right)$ exist (e.g., Tacconi et al. 1994; Sternberg et al. 1994; Usero et al. 2004; Krips et al. 2008; Graciá-Carpio et al. 2008).

Based on the new HCN observations presented in this work and the use of standard conversion factors, we derive values for $\mathrm{SFR}_{\mathrm{ff}}$ and/or $n_{\mathrm{HCN}}\left(\mathrm{H}_{2}\right)$ that are well below the theoretical expectations both for normal galaxies and LIRGs/ULIRGs. Furthermore, the different densities required to fit the two populations of galaxies with a common constant $\mathrm{SFR}_{\mathrm{ff}} \sim 0.02$ are far from the values derived in observations.

\subsubsection{Case-II: revised conversion factors}

Figure $11 \mathrm{~b}$ is similar to Fig. 11a but here $\mathrm{SFR}_{\mathrm{ff}}$ values have been obtained from the revised estimates of the conversion factors discussed in Sect. 6 for $\Sigma_{\text {dense }}$ (in LIRGs/ULIRGs) and $\Sigma_{\text {SFR }}$ (in normal galaxies). Not surprisingly, by adopting the revised conversion factors the global distribution of $\mathrm{SFR}_{\mathrm{ff}}$ is shifted upward and, at the same time, the difference between normal galaxies and LIRGs/ULIRGs is increased. Most of the LIRGs/ULIRGs in Fig. 11b show now values of $\mathrm{SFR}_{\mathrm{ff}}$ that lie within the range predicted by Krumholz \& McKee (2005) with HCN densities very close to $n_{\mathrm{HCN}}^{\text {crit }}$. The problem persists in the fit of normal galaxies, however, where the predicted values for $\mathrm{SFR}_{\mathrm{ff}}$ and/or $n_{\mathrm{HCN}}\left(\mathrm{H}_{2}\right)$ are still well below the expected range. The paradigm of a common constant $\mathrm{SFR}_{\mathrm{ff}}$ in all galaxies can only be saved if we allow this parameter to be as low as $\sim 0.0035$. In this scenario the HCN densities fitting the observed efficiencies would range from $n_{\mathrm{HCN}}^{\text {crit }}$ in normal galaxies to $\sim 10^{6} \mathrm{~cm}^{-3}$ in LIRGs/ULIRGs. 
The fit in densities nevertheless requires us to assume a value for $\mathrm{SFR}_{\mathrm{ff}}$ that is a factor of 5-6 lower than expected by Krumholz \& McKee' s models or derived from CO observations (Krumholz $\&$ Tan 2007).

We can therefore conclude that the paradigm of a constant $\mathrm{SFR}_{\mathrm{ff}}$ predicted by models within the range covered by the new HCN observations of normal galaxies and LIRGs/ULIRGs is not supported. The use of revised conversion factors alleviates the problem especially in LIRGs/ULIRGs. However, within the framework of local models it is not possible to fit the observed differences in the $\mathrm{SFE}_{\text {dense }}$ between normal galaxies and LIRGs/ULIRGs using a common constant $S F R_{\mathrm{ff}} \sim 0.02$ and a set of physically acceptable HCN densities.

\subsection{Observations vs. models: the $L_{F I R} / L^{\prime}{ }_{H C N(1-0)}$ luminosity ratio}

Based on the ISM model developed by Krumholz \& McKee (2005), Krumholz \& Thompson (2007) predicted how the $L_{\mathrm{FIR}} / L_{\mathrm{HCN}(1-0)}^{\prime}$ luminosity ratios should change as a function of the galaxy mean gas density $\bar{n}$ for three different object classes: normal galaxies, intermediate galaxies and starbursts. In Krumholz \& Thompson's formulation, molecular clouds in normal galaxies are characterized by low kinetic temperatures $\left(T_{\mathrm{K}}=10 \mathrm{~K}\right)$, as well as moderate-to-low Mach numbers $(\mathcal{M}=$ $30)$, HCN abundances $\left(X(\mathrm{HCN})=1 \times 10^{-8}\right)$, and $\mathrm{HCN}(1-0)$ opacities $\left(\tau_{\mathrm{HCN}}=0.5\right)$. These molecular cloud parameters are expected to be boosted to higher values in starburst galaxies: $T_{\mathrm{K}}=50 \mathrm{~K}, \mathcal{M}=80, X(\mathrm{HCN})=4 \times 10^{-8}, \tau_{\mathrm{HCN}}=2(\mathrm{see}$ Table 1 of Krumholz \& Thompson 2007, for details). The common envelope to normal and starburst systems is that molecular clouds are characterized by a roughly constant dimensionless star-formation efficiency measured by $\mathrm{SFR}_{\mathrm{ff}}$.

One key prediction of this model is that for mean densities $\bar{n} \leq 10^{4} \mathrm{~cm}^{-3}$, normal galaxies are expected to show higher $\mathrm{SFE}_{\text {dense }}$ than galaxies classified as starbursts. Another key prediction is that the $L_{\mathrm{FIR}} / L_{\mathrm{HCN}(1-0)}^{\prime}$ luminosity ratio should remain roughly constant in a diagram that simultaneously contains data from normal galaxies (characterized by low $\bar{n}$ ) and starburst galaxies (characterized by high $\bar{n}$ ) up to densities $\bar{n} \leq 10^{4} \mathrm{~cm}^{-3}$. A different (less flat) appearance of $\mathrm{SFE}_{\text {dense }}$ as a function of $\bar{n}$ can be predicted at higher densities, however. Figure 12, a modified version of Fig. 2 of Krumholz \& Thompson (2007), purposely explores the predicted $\mathrm{SFE}_{\text {dense }}$ in the high-density regime: $10^{4} \mathrm{~cm}^{-3}<\bar{n}<10^{7} \mathrm{~cm}^{-3}$. Following the convention of Krumholz \& Thompson (2007), we represent the $L_{\mathrm{FIR}} / L_{\mathrm{HCN}(1-0)}^{\prime}$ and $\mathrm{SFR} / L_{\mathrm{HCN}(1-0)}^{\prime}$ ratios as a function of the mean gas density $\bar{n}$ for normal (dot-dashed line), intermediate (solid line) and starburst (dashed line) galaxies ${ }^{4}$. As argued by Krumholz \& Thompson (2007), the models for normal galaxies and starbursts are seen to converge in the high-density regime $\left(\bar{n} \geq 10^{5} \mathrm{~cm}^{-3}\right)$.

As discussed in Sect. 4.1, the observations presented in this paper show that the $L_{\mathrm{FIR}} / L_{\mathrm{HCN}(1-0)}^{\prime}$ ratio is not constant inside our combined sample: $S_{\text {fense }}$ is a factor $\sim 2-3$ higher in LIRGs/ULIRGs compared to normal galaxies $\quad\left(\left\langle\mathrm{SFE}_{\text {dense }}\right\rangle(\mathrm{LIRGs} / \mathrm{ULIRGs}) \sim 1400 \pm 100 L_{\odot} L^{\prime-1}\right.$; $\left\langle\mathrm{SFE}_{\text {dense }}\right\rangle($ normal $\left.) \sim 600 \pm 70 L_{\odot} L^{\prime-1}\right)$. These data can be used to benchmark the predictions of the model shown in Fig. 12. Two conclusions can be drawn from this comparison. First, in

\footnotetext{
4 This figure has been produced using the code of the model made publicly available by Mark Krumholz at http://www. ucolick.org/ ikrumholz/
}

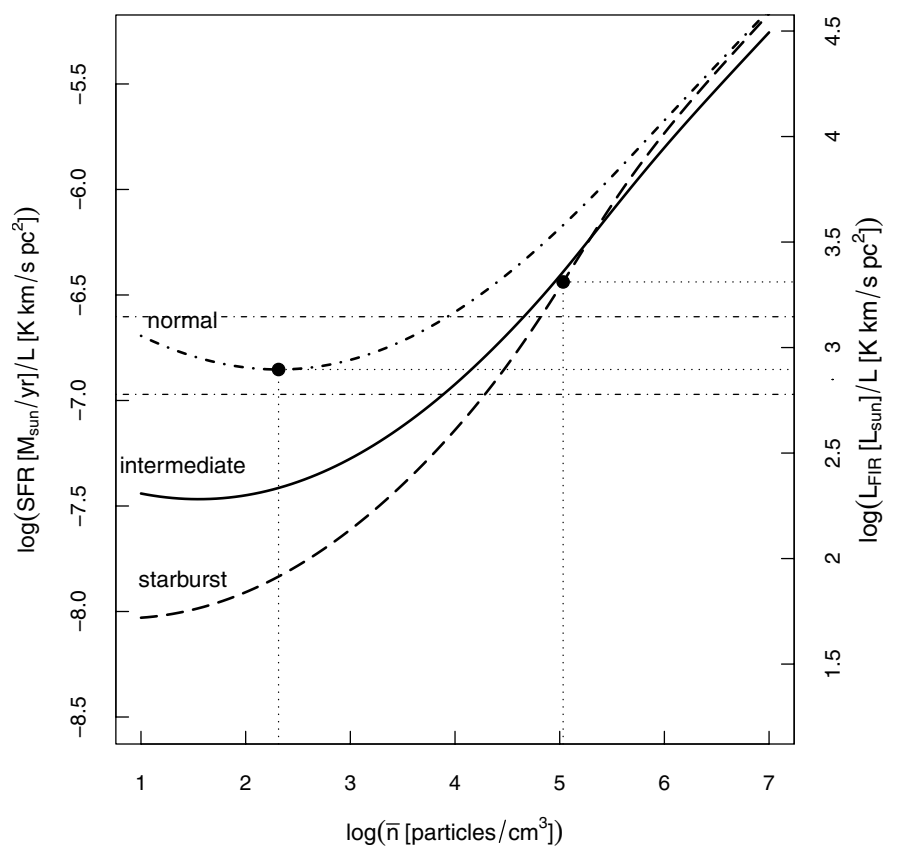

Fig. 12. We show the model-predicted ratio of the star-formation rate or IR luminosity to the $\mathrm{HCN}(1-0)$ luminosity as a function of the mean gas density $\bar{n}$ for the three cases examined by Krumholz \& Thompson (2007): normal (dot-dashed line), intermediate (solid line) and starburst (dashed line) galaxies. The dot-dashed horizontal lines highlight the $L_{\mathrm{FIR}} / L_{\mathrm{HCN}(1-0)}^{\prime}$ observed in normal galaxies and LIRGs/ULIRGs. The vertical dot lines indicate the location of the best agreement between observations and models as discussed in the text.

relative terms, the model is able to reproduce the increase in the $\mathrm{SFE}_{\text {dense }}$ derived from observations as being caused by a dramatic increase in the average gas densities. According to Fig. 12, if we assume that normal galaxies are characterized by $\bar{n}=2 \times 10^{2} \mathrm{~cm}^{-3}$, extreme starbursts (LIRGs/ULIRGs) should be in a $\bar{n}=10^{5} \mathrm{~cm}^{-3}$ regime to fit the observed change in $\mathrm{SFE}_{\mathrm{dense}}$. In absolute terms, the model predicts values of $\mathrm{SFE}_{\mathrm{dense}}$ for normal galaxies and extreme starbursts that are marginally a factor $\sim 1.5$ above the observed values ${ }^{5}$. This success of the model would be in apparent contradiction with the scenario described in the previous section, where we concluded that the star-formation efficiency per free-fall time SFR $_{\mathrm{ff}}$ in the model, supposed to be $\sim 0.02$, has to be lowered by a factor 5-6 to fit the observations (after the revision of conversion factors discussed in Sect. 7.1.2). As argued below, there is no contradiction, however. Based on the output of the model of Krumholz \& Thompson, we can estimate the implicit value of $\alpha^{\mathrm{HCN}}$ for the model molecular clouds at densities higher than $n_{\mathrm{HCN}}^{\text {crit }}=3 \times 10^{4} \mathrm{~cm}^{-3}$. The HCN conversion factor depends, for each galaxy class, on $\bar{n}$. Assuming the best fit values for $\bar{n}$ reported above, we derive that $\alpha^{\mathrm{HCN}} \sim 1-2 M_{\odot} L^{\prime-1}$ in starbursts and $\sim 3-4 M_{\odot} L^{\prime-1}$ in normal galaxies in the model, i.e., a factor $\sim 2-3$ lower than the values of $\alpha^{\mathrm{HCN}}$ adopted in Sect. 6.1. Taking into account that the best compromise solution for $L_{\mathrm{FIR}} / L_{\mathrm{HCN}(1-0)}^{\prime}$ implies ratios that are still $\sim 50 \%$ too high

5 We note that the absolute scale along the right $Y$ axis of Fig. 2 of Krumholz \& Thompson (2007) seems to be incorrect. A re-evaluation of this scale, derived from the output of the model, indicates that the predicted $L_{\mathrm{FIR}} / L_{\mathrm{HCN}(1-0)}^{\prime}$ ratios are a factor of 2-3 lower than shown in the figure. Of particular note, this change helps finding a better agreement between observations and the model. 
(Fig. 12), we conclude that the model (with $S F R_{\mathrm{ff}} \sim 0.02$ and our preferred values for $\alpha^{\mathrm{HCN}}$ ) is a factor of $\sim 4$ off. This is similar to the factor of $\sim 5-6$ disagreement found in Sect. 7.1.2.

The conclusion common to the present section and Sect. 7.1 is that a rough agreement between the HCN observations presented in this paper and the predictions of local models can only be reached if SFR $\mathrm{ff}_{0} \sim 0.005-0.01$ and/or if $\alpha^{\mathrm{HCN}}$ is still a factor of $\sim$ a few lower than our favored values for normal galaxies and starbursts (LIRGs/ULIRGs). In qualitative agreement with this picture, the recent paper of Krumholz et al. (2012) has presented conclusive evidence that the best correspondence between the prediction of local models and the results of $\mathrm{CO}$ galaxy surveys requires lowering $\mathrm{SFR}_{\mathrm{ff}}$ to 0.01 , i.e., a factor of 1.5-3 below the value previously assumed by Krumholz \& McKee (2005). Dib (2011) recently came to similar conclusions that also point to lower values of $\mathrm{SFR}_{\mathrm{ff}}$ based on a different formulation of the star-formation process, which is regulated in his model by stellar feedback.

\subsection{Observations vs. models: is "global" dynamical time scale a key parameter?}

We have provided evidence that the conversion of dense molecular gas into stars is more efficient in LIRGs/ULIRGs than in normal galaxies. Although this has been ascribed to the existence of very different local physical properties (densities) of the clouds in these two populations of galaxies, we have seen in Sects. 7.1 and 7.2 that this hypothesis has difficulties in explaining the HCN results discussed in this paper (unless significantly lower $\mathrm{SFR}_{\mathrm{ff}}$ and/or $\alpha^{\mathrm{HCN}}$ are assumed). Alternatively, the starformation rates could be regulated by the global large-scale dynamics of the ISM, more perturbed in strongly interacting galaxies (in our sample most of them are LIRGs/ULIRGs) than in normal galaxies. In this scenario we expect that the differences in $\mathrm{SFE}_{\text {dense }}$ (or equivalently in $t_{\mathrm{dep}}$ ) between the two populations of galaxies can be reproduced by similar differences in $t_{\mathrm{dyn}}$, as $\mathrm{SFE}_{\text {dense }} \equiv 1 / t_{\mathrm{dep}} \propto 1 / t_{\mathrm{dyn}}$. To explore this possibility, we compare in this section the distribution of dynamical time scales and depletion time scales of the dense molecular gas in our sample.

Figure 13 represents $\log \left(t_{\mathrm{dep}}\right)$ versus $\log \left(t_{\mathrm{dyn}}\right)$ for normal galaxies and LIRGs/ULIRGs. Depletion time scales of the dense gas $t_{\text {dep }}$ are derived as defined in Sect. 4.1. Dynamical time scales $t_{\text {dyn }}$ are defined as a crossing time scale unit determined by the ratio between the radius of the star-forming region, where the $\mathrm{HCN}$ emitting gas is expected to lie, and half the full width of the $\mathrm{HCN}$ line. When available, we corrected $t_{\text {dyn }}$ for the value of the inclination of the galaxies. Whereas there is a clear segregation between the two populations of galaxies as to the values of $t_{\text {dep }}$, the corresponding differences in $t_{\text {dyn }}$ are less significant.

Figure 14 shows a histogram representation of the number density of galaxies as a function of $t_{\mathrm{dep}}, t_{\mathrm{dyn}}$ and the $t_{\text {dep }} / t_{\text {dyn }}$ ratio. We split our sample into normal galaxies $\left(L_{\mathrm{IR}}<\right.$ $10^{11} L_{\odot}$, gray filled histograms) and luminous infrared galaxies $\left(L_{\mathrm{IR}}>10^{11} L_{\odot}\right.$, empty histograms). Depletion times (top panel) roughly span an order of magnitude in each subsample. They peak around $\sim 10 \mathrm{Myr}$ for LIRGs/ULIRGs and around $\sim 50$ Myr for normal galaxies. The clear separation between the two histograms highlights the higher star-formation efficiency of LIRGs/ULIRGs. The middle panel shows the dynamical time scales of the studied galaxies. The two histograms extend across a common range of $\sim 1.5$ orders of magnitude, although the dynamical time scales in LIRGs/ULIRGs are about a factor of 2 lower on average. These differences between the

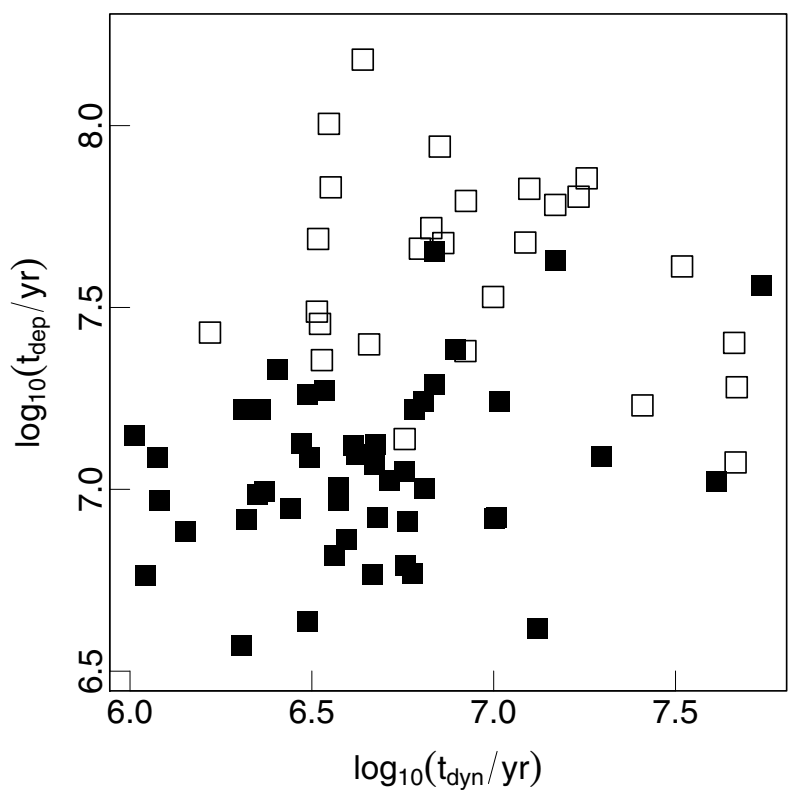

Fig. 13. We represent $\log \left(t_{\text {dep }}\right)$ versus $\log \left(t_{\text {dyn }}\right)$ for normal galaxies $\left(L_{\mathrm{IR}}<10^{11} L_{\odot}\right.$, open symbols) and luminous infrared galaxies $\left(L_{\mathrm{IR}}>\right.$ $10^{11} L_{\odot}$, filled symbols).

two populations of galaxies can be expected, because many LIRGs/ULIRGs are mergers where the interaction has brought most of the molecular gas to smaller radii. We note, however, that the differences reported for $t_{\mathrm{dyn}}$ fall short of explaining the factor 5 difference in $t_{\text {dep }}$.

We represent in the lower panel of Fig. 14 the histograms of the $t_{\mathrm{dep}} / t_{\mathrm{dyn}}$ ratio. This is equivalent to the ratio between the mass of dense molecular gas and the mass of stars formed in one dynamical time scale. Although the shape (intrinsic dispersion) of the two histograms is similar, $t_{\text {dep }} / t_{\text {dyn }}$ ratios in LIRGs/ULIRGs are typically a factor of 2 lower than in normal galaxies. Taken at face value, this suggests that the amount of dense gas converted into stars per unit of dynamical time is somewhat higher in LIRGs/ULIRGs. This result would contradict the idea that star formation is governed mainly by global dynamical time scales, because in this case we expect that the $t_{\text {dep }} / t_{\text {dyn }}$ ratio is roughly constant, and consequently shows no significant differences between the two galaxy populations.

The inability of "global" star-formation models to fully explain the KS law derived from HCN, anticipated above, is better illustrated in Fig. 15. We show in this figure the threedimensional representation of the bi-parametric star-formation law that expresses the star-formation rate density $\Sigma_{\mathrm{SFR}}$ as a function of the dense molecular gas surface density $\Sigma_{\text {dense }}$ and the dynamical time $t_{\text {dyn }}$. The best fit to the HCN data is given by

$\log \Sigma_{\mathrm{SFR}}=(0.8 \pm 0.1) \log \Sigma_{\text {dense }}+(-0.5 \pm 0.2) \log t_{\text {dyn }}+(2.6 \mp 1)$.

While the power law index for $\Sigma_{\text {dense }}(0.8 \pm 0.1)$ is not far from unity within the errors, the corresponding index for $t_{\mathrm{dyn}}$ $(-0.5 \pm 0.2)$ is not compatible with -1 . This is against the theoretical expected power law index for $\Sigma_{\text {dense }} / t_{\text {dyn }}$ ratio that should be about $\sim 1$ (Kennicutt 1998). Furthermore, the goodness of the fit of Eq. (21), estimated by a standard $\chi^{2}$ analysis, indicates a meager $12 \%$ improvement with respect to the value of $\chi^{2}$ obtained in the single parameter fit of Eq. (19). With the inclusion of "global" dynamical time scales we are unable to significantly improve the fit of star-formation laws derived from $\mathrm{HCN}$. 

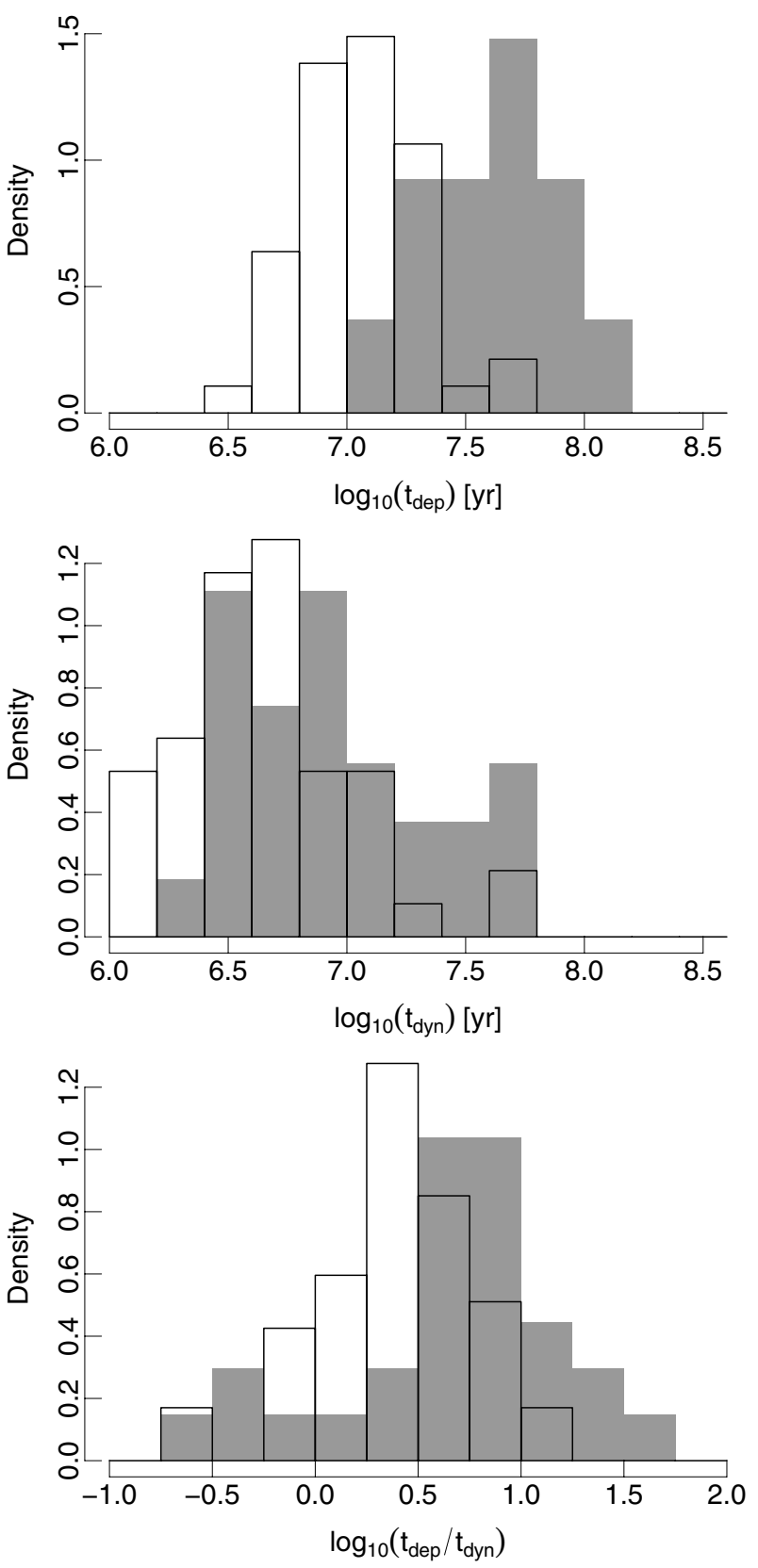

Fig. 14. a) (Upper panel) Histograms showing the number density of galaxies (number of galaxies per unit interval, as shown) as a function of $\log \left(t_{\mathrm{dep}}\right)$ for normal galaxies $\left(L_{\mathrm{IR}}<10^{11} L_{\odot}\right.$, gray filled histogram) and luminous infrared galaxies $\left(L_{\mathrm{IR}}>10^{11} L_{\odot}\right.$, empty histogram). b) (Middle panel) Same as a) but representing the number density of galaxies as a function of $\log \left(t_{\mathrm{dyn}}\right)$. c) (Lower panel) Same as a) but representing the number density of galaxies as a function of $\log \left(t_{\mathrm{dep}} / t_{\mathrm{dyn}}\right)$.

Several biases in the way the dynamical time scales are derived could explain why the $t_{\text {dep }} / t_{\text {dyn }}$ histograms shown in Fig. 14 are not coincident for the two populations of galaxies. In particular, dynamical time scales in normal galaxies, where star formation is typically much more widely spread in the disks compared to LIRG/ULIRGs, could be underestimated. However, LIRGs/ULIRGs do not behave as a monolithic family in this respect: some LIRGs, especially in the range $L_{\mathrm{IR}} \leq$ $10^{11.4} L_{\odot}$ can have spread-out star formation (Hattori et al. 2004; Alonso-Herrero et al. 2006; Rodríguez-Zahurín et al. 2011).

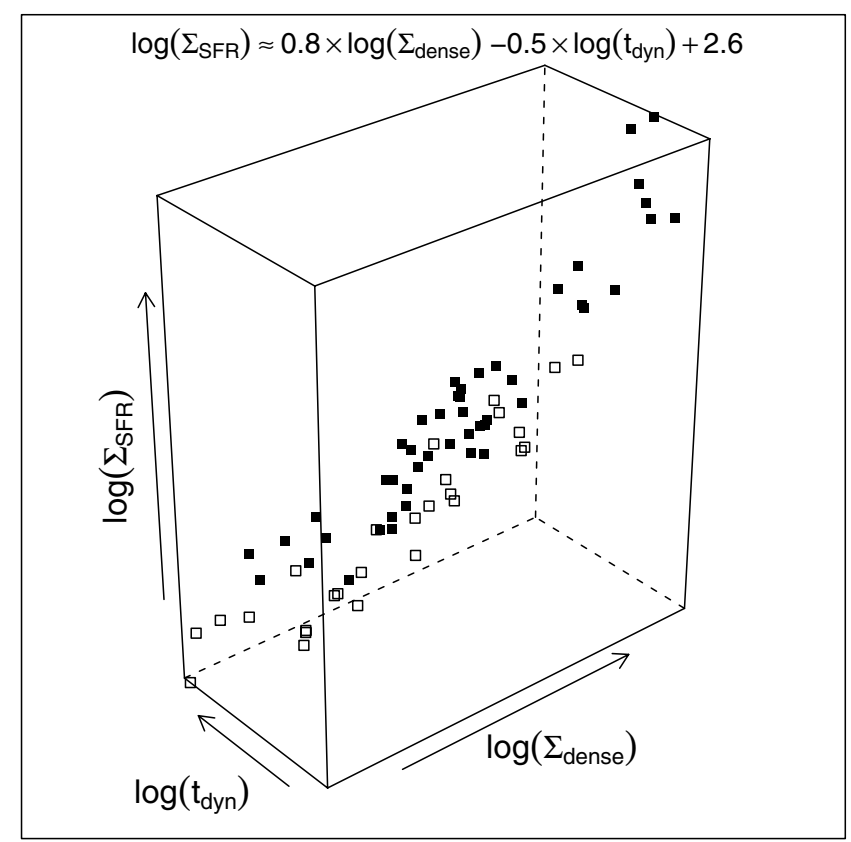

Fig. 15. Three-dimensional representation of the bi-parametric starformation law giving the star-formation rate density $\Sigma_{\text {SFR }}$ as a function of the dense molecular gas surface density $\Sigma_{\text {dense }}$ and the dynamical time $t_{\mathrm{dyn}}$.

\section{Summary and conclusions}

We have used the IRAM 30 m telescope to observe a sample of 19 LIRGs in the 1-0 lines of $\mathrm{CO}, \mathrm{HCN}$ and $\mathrm{HCO}^{+}$. The galaxies were extracted from a sample of local LIRGs with available high-quality and high-resolution images obtained at optical, near- and mid-IR wavelengths, which probe the star-formation activity. The new data presented in this work allow us to expand the number of LIRGs studied in $\mathrm{HCN}-\mathrm{HCO}^{+}$lines by more than a factor 3 compared to previous works. The chosen LIRG sample has a range of $\mathrm{HCN}$ luminosities that overlaps to a significant extent with that of normal galaxies. This is a key requirement for testing if LIRGs represent a transition point in star-formation laws between normal galaxies and ULIRGs. In particular, we studied if the observed bimodality of star-formation laws in galaxies derived from $\mathrm{CO}$ line data in previous surveys can be extended to the higher density regime probed by HCN. We used these results to explore the validity of different theoretical prescriptions for star-formation laws where local and/or global dynamical time scales are explicitly included.

We summarize below the main results and conclusions of this work:

- We find that the star-formation efficiency of the dense gas ( $\mathrm{SFE}_{\text {dense }}$ ) estimated by $L_{\mathrm{FIR}} / L_{\mathrm{HCN}(1-0)}^{\prime}$ luminosity ratios is on average a factor $\sim 2-3$ higher in LIRGs/ULIRGs $\left(\sim 1400 \pm 100 L_{\odot} L^{\prime-1}\right) \quad$ compared to normal galaxies $\left(\sim 600 \pm 70 L_{\odot} L^{\prime-1}\right)$. With the addition of the new LIRG sample we confirm on a more solid statistical basis that the $L_{\mathrm{FIR}}-L_{\mathrm{HCN}(1-0)}^{\prime}$ correlation is significantly superlinear (power law $\sim 1.23 \pm 0.05)$ when we include normal galaxies and LIRGs/ULIRGs in the fit.

- We derived KS laws relating the two surface densities, $\Sigma_{\text {SFR }}$ and $\Sigma_{\text {dense }}$ in normal galaxies and LIRGs/ULIRGs. A fit to the full sample yielded a power index $N=1.12 \pm 0.04$. 
However, LIRGs/ULIRGs and normal galaxies are not fully overlapping in this scatter plot. In order to quantify if a dual fit applies to the power law, we split the sample into normal and IR luminous galaxies. While the $\left(\Sigma_{\mathrm{SFR}}, \Sigma_{\text {dense }}\right)$ distribution cannot be described as strictly bimodal, we found that a two-function power law fit with indices close to unity qualifies as a much better description of the star-formation relation for the dense gas. This result is seen to be solid against the statistical biases inherent to this analysis.

- If we account for the different conversion factors for $\mathrm{HCN}$ in extreme starbursts and for the unobscured star-formation rate in normal galaxies, for which we find new evidence in these observations, the duality in star-formation laws is enhanced. This result extends the more extreme bimodal behavior of star-formation laws, derived from $\mathrm{CO}$ molecular lines by two recent surveys (Daddi et al. 2010; Genzel et al. 2010), to the higher molecular densities probed by HCN lines. The revised depletion time scales for the dense molecular gas show a significant difference between LIRGs/ULIRGs $\left(t_{\text {dep }} \sim 14 \pm 1.4 \mathrm{Myr}\right)$ and normal galaxies $\left(t_{\text {dep }} \sim 50 \pm 5 \mathrm{Myr}\right)$.

- We compared the results of the new HCN observations with the predictions of theoretical models in which the efficiency of star formation is determined by the ratio of a constant star-formation rate per free-fall time $\left(\mathrm{SFR}_{\mathrm{ff}}\right)$ to the local freefall time $\left(t_{\mathrm{ff}}\right)$ (Krumholz \&McKee 2005). Within the framework of these models we found that it is possible to fit the observed differences in the $\mathrm{SFE}_{\text {dense }}$ between normal galaxies and LIRGs/ULIRGs using a common constant SFR $_{\mathrm{ff}}$ and a set of physically acceptable $\mathrm{HCN}$ densities, but only if $\mathrm{SFR}_{\mathrm{ff}} \sim 0.005-0.01$ and/or if $\alpha^{\mathrm{HCN}}$ is still a factor of $\sim$ a few lower than our favored values.

- We studied whether the inclusion of "global" dynamical time scales offers a better description of the star-formation relations derived for the dense gas. This approach helps finding a marginally better universal relation for the dense molecular gas. In the best-fit solution for $\Sigma_{\mathrm{SFR}} \propto \Sigma_{\text {dense }}^{n} t_{\text {dyn }}^{m}$, the power law index for $\Sigma_{\text {dense }}(n=0.8 \pm 0.1)$ is not far from unity within the errors, i.e., in agreement with the theoretical expected value. Nevertheless, the corresponding index for $t_{\text {dyn }}$ ( $m=-0.5 \pm 0.2$ ) is not compatible with -1 , a result whose theoretical implications remain to be understood.

A fit of the SF laws similar to the one discussed in Sect. 7.3 was first explored by Genzel et al. (2010), who used CO line data in a sample of normal SF galaxies and extreme starbursts at different redshift ranges. The two-parameter $\left(M_{\mathrm{gas}}, t_{\mathrm{dyn}}\right)$ SFR law derived by Genzel et al. (2010) (SFR $\left.\propto M_{\text {gas }}^{1.4} t_{\text {dyn }}^{-0.8}\right)$ is successful at providing a universal SF relation. Remarkably, the power law indices in their fitted SF relation are also found to deviate from the theoretical expected values ( $\mathrm{SFR} \propto M_{\mathrm{gas}}^{1} t_{\mathrm{dyn}}^{-1}$ ), however. Altogether the SF relation obtained in Eq. (21) from $\mathrm{HCN}$ data and the one obtained by Genzel et al. (2010) from CO are very similar to the KS laws obtained, respectively, by Gao \& Solomon (2004b) and Kennicutt (1998) provided that we remain in the $\Sigma_{\mathrm{SFR}}-\Sigma_{(\mathrm{dense}) \mathrm{gas}}$ projection of the SF laws.

The bottom line, however, is that the different dynamical time scale ranges found for normal galaxies and extreme starbursts are not sufficient to dissolve the duality observed in the $\Sigma_{\text {SFR }}-\Sigma_{\text {(dense)gas }}$ projection of the SF laws (see also Krumholz et al. 2012). A possible explanation for this failure can be searched for in the uncertainties that plague the definition and the observational determination of $t_{\text {dyn }}$. This is paramount if we are to compare systems that are known to be in very different dynamical states. So far, the most widely accepted definition for $t_{\text {dyn }}$, also used in this work, is equivalent to that of a crossing or orbit time scale for the system, which is determined from observations. However, while normal galaxies can be described as dynamically relaxed rotating disks, mergers are strongly interacting and dynamically very disturbed. In this context using a common definition for $t_{\text {dyn }}$ is questionable. High-spatial resolution observations can help to disentangle the role of largescale dynamics in the small-scale spatially resolved SF laws both in normal galaxies and in extreme starbursts (e.g. Boquien et al. 2011).

Alternatively, the inclusion of global dynamical time scales may not be the key for estimating the correct scaling of the SF relation and thus for determining the absolute value of the SFR for different galaxy populations. From the observational point of view there is ample room for improvement in our understanding of how conversion factors for different tracers of molecular gas may differ in galaxies. As discussed above, this is a prerequisite for accurately calibrating SF relations and exploring their differences between galaxies. So far, the evidence for different conversion factors in normal galaxies and extreme starbursts is limited to $\mathrm{CO}$ and $\mathrm{HCN}$ lines. Multi-transition studies for different molecular tracers will be paramount for tackling this problem.

In addition to providing a better handle of conversion factors, which are required to convert intensities $(I)$ into gas surface densities $\left(\Sigma_{\text {gas }}\right)$, multi-line observations can yield an estimate of volume gas densities $\left(\rho_{\text {gas }}\right)$. This will facilitate a more direct comparison between observations and models that predict how the SFR volume density should depend on $\rho_{\text {gas }}$, rather than on $\Sigma_{\text {gas }}$. In this context we mention that Krumholz et al. (2012) have recently argued that a simple, local, volumetric star-formation law with $\mathrm{SFR}_{\mathrm{ff}} \sim 0.01$ can dissolve the apparent bimodality found by Genzel et al. (2010) if different scale-heights are assumed for the gas disks $\left(h_{\text {gas }}\right)$ in normal galaxies and extreme starbursts. While this is a plausible explanation, we note that the observational constraints on how $h_{\text {gas }}$ might differ in different galaxy populations are currently scarce. The change in $h_{\text {gas }}$ required to make duality/bimodality disappear in SF laws consequently remains to be observationally validated.

Acknowledgements. We acknowledge the IRAM staff at $30 \mathrm{~m}$ telescope for their help during the observations data reduction. S.G.B. acknowledges support from MICIN within program CONSOLIDER INGENIO 2010, under grant "Molecular Astrophysics: The Herschel and ALMA Era-ASTROMOL" (Ref. CSD2009-00038). A.A.H., M.P.S., L.C. and S.A. acknowledge support from AYA2010-21161-C02-1. M.P.S. also acknowledges support from the CSIC under grant JAE-Predoc-2007. We also thank the anonymous referee for his/her insightful and constructive report.

\section{References}

Aalto, S., Booth, R. S., Black, J. H., \& Johansson, L. E. B. 1995, A\&A, 300, 369 Alonso-Herrero, A., Engelbracht, C. W., Rieke, M. J., Rieke, G. H., \& Quillen, A. C. 2001, ApJ, 546, 952

Alonso-Herrero, A., Rieke, G. H., Rieke, M. J., \& Scoville, N. Z. 2002, AJ, 124, 166

Alonso-Herrero, A., Rieke, G. H., Rieke, M. J., et al. 2006, ApJ, 650, 835

Alonso-Herrero, A., García-Marín, M., Monreal-Ibero, A., et al. 2009, A\&A, 506,1541

Alonso-Herrero, A., Pereira-Santaella, M., Rieke, G. H., \& Rigopoulou, D. 2012, ApJ, in press [arXiv: 1109.1372]

Armus, L., Mazzarella, J. M., Evans, A. S., et al. 2009, PASP, 121, 559

Bayet, E., Gerin, M., Phillips, T. G., \& Contursi, A. 2009, MNRAS, 399, 264

Bigiel, F., Leroy, A., Walter, F., et al. 2008, AJ, 136, 2846

Boissier, S., Prantzos, N., Boselli, A., \& Gavazzi, G. 2003, MNRAS, 346, 1215

Boquien, M., Lisenfeld, U., Duc, P.-A., et al. 2011, A\&A, 533, A19

Bouché, N., Cresci, G., Davies, R., et al. 2007, ApJ, 671, 303

Bussmann, R. S., Narayanan, D., Shirley, Y. L., et al. 2008, ApJ, 681, L73

Calzetti, D., Kennicutt, R. C., Engelbracht, C. W., et al. 2007, ApJ, 666, 870

Combes, F. 1991, ARA\&A, 29, 195

Daddi, E., Elbaz, D., Walter, F., et al. 2010, ApJ, 714, L118 
Dib, S. 2011, ApJ, 737, L20

Downes, D., \& Solomon, P. M. 1998, ApJ, 507, 615

Elmegreen, B. G. 1997, Rev. Mex. Astron. Astrofis. Conf. Ser., 6, 165

Elmegreen, B. G. 2002, ApJ, 577, 206

Evans, A. S., Solomon, P. M., Tacconi, L. J., Vavilkin, T., \& Downes, D. 2006, AJ, 132, 2398

Gao, Y., \& Solomon, P. M. 2004a, ApJS, 152, 63

Gao, Y., \& Solomon, P. M. 2004b, ApJ, 606, 271

Gao, Y., Carilli, C. L., Solomon, P. M., \& Vanden Bout, P. A. 2007, ApJ, 660, L93

García-Burillo, S., Graciá-Carpio, J., Guélin, M., et al. 2006, ApJ, 645, L17

García-Marín, M., Colina, L., \& Arribas, S. 2009, A\&A, 505, 1017

Genzel, R., Tacconi, L. J., Gracia-Carpio, J., et al. 2010, MNRAS, 407, 2091

Graciá-Carpio, J., García-Burillo, S., Planesas, P., \& Colina, L. 2006, ApJ, 640, L135

Graciá-Carpio, J., García-Burillo, S., Planesas, P., et al. 2008, A\&A, 479

Graciá-Carpio, J., Sturm, E., Hailey-Dunsheath, S., et al. 2011, ApJ, 728, L7

Guélin, M., Salomé, P., Neri, R., et al. 2007, A\&A, 462, L45

Hartigan, J. A., \& Hartigan, P. M. 1985, Ann. Stat., 13, 70

Hattori, T., Yoshida, M., Ohtani, H., et al. 2004, AJ, 127, 736

Juneau, S., Narayanan, D. T., Moustakas, J., et al. 2009, ApJ, 707, 1217

Kennicutt, R. C., Jr. 1998, ApJ, 498, 541

Kennicutt, R. C., Hao, C.-N., Calzetti, D., et al. 2009, ApJ, 703, 1672

Krips, M., Neri, R., García-Burillo, S., et al. 2008, ApJ, 677, 262

Krumholz, M. R., \& McKee, C. F. 2005, ApJ, 630, 250

Krumholz, M. R., \& Tan, J. C. 2007, ApJ, 654, 304

Krumholz, M. R., \& Thompson, T. A. 2007, ApJ, 669, 289

Krumholz, M. R., McKee, C. F., \& Tumlinson, J. 2009, ApJ, 699, 850

Krumholz, M. R., Dekel, A., \& McKee, C. F. 2012, ApJ, 745, 69

Leroy, A. K., Walter, F., Brinks, E., et al. 2008, AJ, 136, 2782

Narayanan, D., Groppi, C. E., Kulesa, C. A., \& Walker, C. K. 2005, ApJ, 630, 269
Narayanan, D., Cox, T. J., Shirley, Y., et al. 2008, ApJ, 684, 996

Nguyen, Q.-R., Jackson, J. M., Henkel, C., Truong, B., \& Mauersberger, R. 1992, ApJ, 399, 521

Pérez-González, P. G., Kennicutt, R. C., Jr., Gordon, K. D., et al. 2006, ApJ, 648, 987

Riechers, D. A., Walter, F., Carilli, C. L., \& Bertoldi, F. 2007, ApJ, 671, L13

Rodríguez-Zaurín, J., Arribas, S., Monreal-Ibero, A., et al. 2011, A\&A, 527, A60 Sanders, D., \& Ishida, C. 2004, The Neutral ISM in Starburst Galaxies (ASP), ed. S. Aalto, S. Huttemeister, \& A. Pedlar, 320, 230

Sanders, D. B., Mazzarella, J. M., Kim, D.-C., et al. 2003, AJ, 126, 1607

Schmidt, M. 1959, ApJ, 129, 243

Scoville, N. Z., Yun, M. S., \& Bryant, P. M. 1997, ApJ, 484, 702

Silk, J. 1997, ApJ, 481, 703

Solomon, P. M., \& Vanden Bout, P. A. 2005, ARA\&A, 43, 677

Solomon, P. M., Downes, D., Radford, S. J. E., \& Barrett, J. W. 1997, ApJ, 478, 144

Sternberg, A., Genzel, R., \& Tacconi, L. 1994, ApJ, 436, L131

Tacconi, L. J., Genzel, R., Blietz, M., et al. 1994, ApJ, 426, L77

Tacconi, L. J., Genzel, R., Smail, I., et al. 2008, ApJ, 680, 246

Tan, J. C. 2010, ApJ, 710, L88

Tasker, E. J., \& Tan, J. C. 2009, ApJ, 700, 358

Toomre, A. 1964, ApJ, 139, 1217

Usero, A., García-Burillo, S., Fuente, A., et al. 2004, A\&A, 419, 897

Lintott, C. J., Viti, S., Williams, D. A., Rawlings, J. M. C., \& Ferreras, I. 2005, MNRAS, 360, 1527

Wagg, J., Wilner, D. J., Neri, R., Downes, D., \& Wiklind, T. 2005, ApJ, 634, L13

Weiß, A., Downes, D., Neri, R., et al. 2007, A\&A, 467, 955

Wild, W., Harris, A. I., Eckart, A., et al. 1992, A\&A, 265, 447

Wu, J., Evans, N. J., II, Gao, Y., et al. 2005, ApJ, 635, L173

Yao, L., Seaquist, E. R., Kuno, N., \& Dunne, L. 2003, ApJ, 588, 771 